\title{
Characterization of Three High Efficiency and Blue Sensitive Silicon Photomultipliers
}

\author{
Adam Nepomuk Otte ${ }^{\mathrm{a}, *}$, Distefano Garcia ${ }^{\mathrm{a}}$, Thanh Nguyen ${ }^{\mathrm{a}}$, Dhruv Purushotham ${ }^{\mathrm{a}}$ \\ ${ }^{a}$ School of Physics 83 Center for Relativistic Astrophysics, Georgia Institute of Technology 837 State Street NW, Atlanta, GA 30332-0430, \\ U.S.A.
}

\begin{abstract}
We report about the optical and electrical characterization of three high efficiency and blue sensitive Silicon photomultipliers from FBK, Hamamatsu, and SensL. Key features of the tested devices when operated at $90 \%$ breakdown probability are peak photon detection efficiencies between $40 \%$ and $55 \%$, temperature dependencies of gain and PDE that are less than $1 \% /{ }^{\circ} \mathrm{C}$, dark rates of $\sim 50 \mathrm{kHz} / \mathrm{mm}^{2}$ at room temperature, afterpulsing of about $2 \%$, and direct optical crosstalk between $6 \%$ and $20 \%$. The characteristics of all three devices impressively demonstrate how the Silicon-photomultiplier technology has improved over the past ten years. It is further demonstrated how the voltage and temperature characteristics of a number of quantities can be parameterized on the basis of physical models. The models provide a deeper understanding of the device characteristics over a wide bias and temperature range. They also serve as examples how producers could provide the characteristics of their SiPMs to users. A standardized parameterization of SiPMs would enable users to find the optimal SiPM for their application and the operating point of SiPMs without having to perform measurements thus significantly reducing design and development cycles.
\end{abstract}

Keywords: Silicon photomultiplier, SiPM, photon detector, characterization, G-APD

\section{Introduction}

Silicon photomultipliers (SiPMs) have attracted signif- ${ }^{27}$ icant attention over the past few years. They are be- ${ }_{29}$ coming increasingly popular in scientific and industrial ${ }_{30}$ applications, which require fast, highly-efficient, singlephoton-resolving photon detectors. Some prominent appli- ${ }_{32}$ cations are in the fields of high-energy physics, astroparticle physics, and medical imaging (s. e.g. $[1,2,3,4])_{34}$ Reasons for the popularity of SiPMs are their high photondetection efficiencies, mechanical and electrical robustness, ${ }_{36}$ low mass, low power, low bias voltages.

Another reason for the increasing popularity of $\mathrm{SiPMs}_{38}{ }_{37}^{37}$ is that in recent years, they have been subject to many improvements. In particular, recent developments have successfully addressed nuisances such as high optical ${ }_{41}^{40}$ crosstalk, high afterpulsing, and high dark rates, but they ${ }_{42}$ have also improved the photon detection efficiency, which ${ }_{43}$ previously limited the usefulness of SiPMs in several ap- ${ }_{44}$ plications.

We are interested in SiPMs because we aim to use them in Cherenkov telescopes to detect gamma rays from astrophysical sources. Cherenkov telescopes image the ${ }^{46}$ Cherenkov light emitted from relativistic particle showers that are initiated by cosmic rays and gamma rays in ${ }^{47}$ the atmosphere [5]. An in-depth understanding of photon ${ }_{49}^{48}$

\footnotetext{
* Corresponding author

Email address: otte@gatech.edu (Adam Nepomuk Otte)
}

Preprint submitted to Elsevier detectors down to the level of device physics is key in the pursuit of minimizing the systematic uncertainties present in the Cherenkov telescope data.

In this paper we present an in-depth and comparative study of three recent, blue-sensitive SiPMs from FBK, SensL, and Hamamatsu, which demonstrate impressive performance improvements compared to devices from only a few years ago, e.g. [6]. Beside the three tested devices many more devices exist from other vendors, which could not be tested due to a lack of time and resources. Along with our results we give a detailed description of our test setups and discuss the measurement procedures and resulting systematic uncertainties. We, furthermore, parameterize the overvoltage and temperature dependencies of most parameters. Where possible we use a physics-motivated model for the parameterization, which allows us to gain further insight into the device physics of SiPMs. We hope that the parameterizations we use will help to further standardize the measurement and parameterization of SiPM characteristics.

\section{Device descriptions}

SiPMs are semiconductor-based photon detectors that consist of a matrix of elementary cells, which are avalanche photodiodes operating in Geiger mode. In the conventional SiPM, which is the type of SiPMs tested here, each cell is connected to a series resistor that limits the current flowing during the breakdown and thus ensures that the 


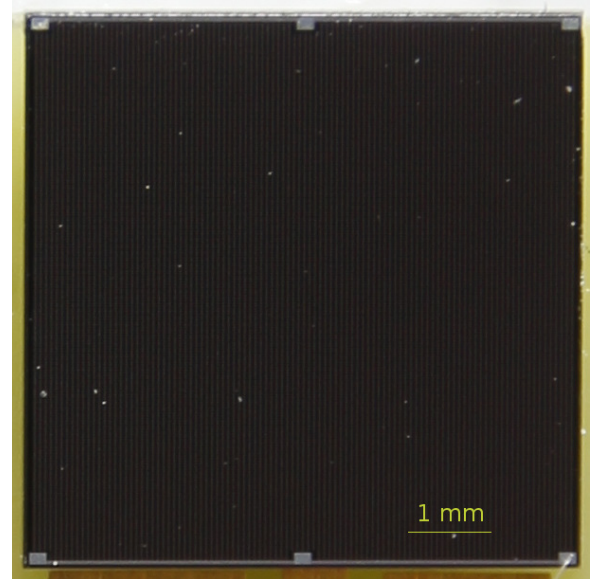

(a) FBK NUV-HD

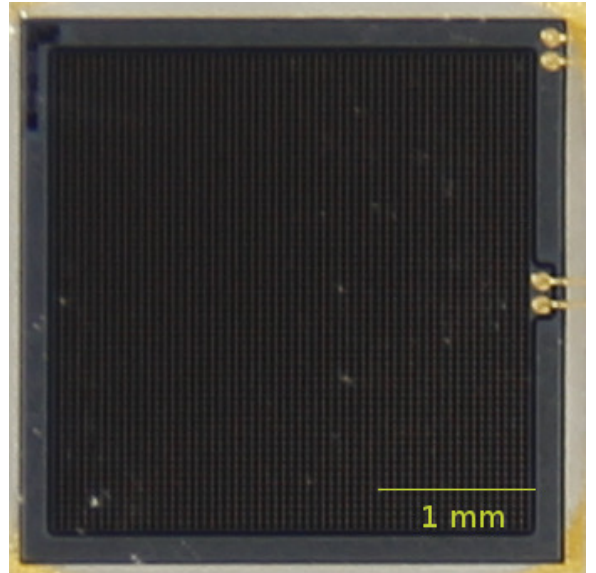

(b) Hamamatsu S13360-3050CS

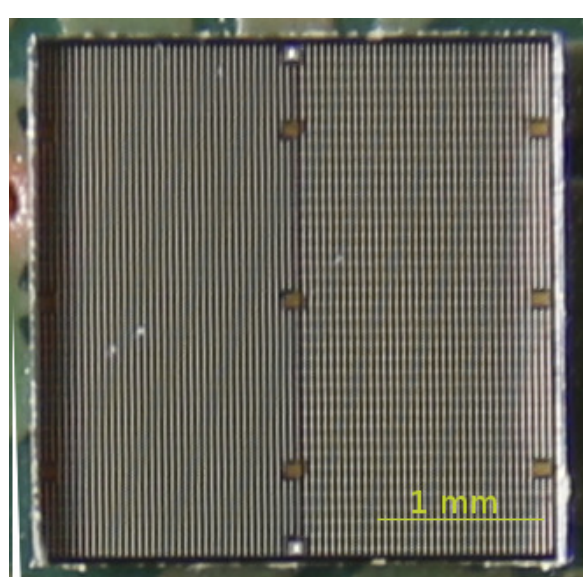

(c) SensL J-series 30035

Figure 1: Full scale pictures of the three tested SiPMs.

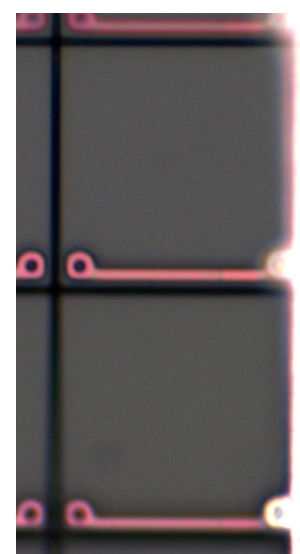

(a) FBK NUV-HD

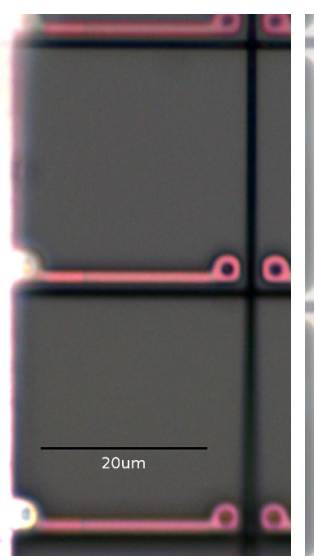

10

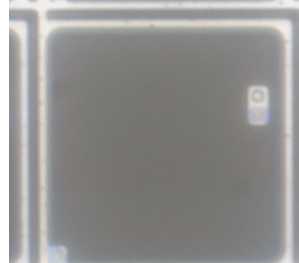

(b) Hamamatsu S13360-3050CS

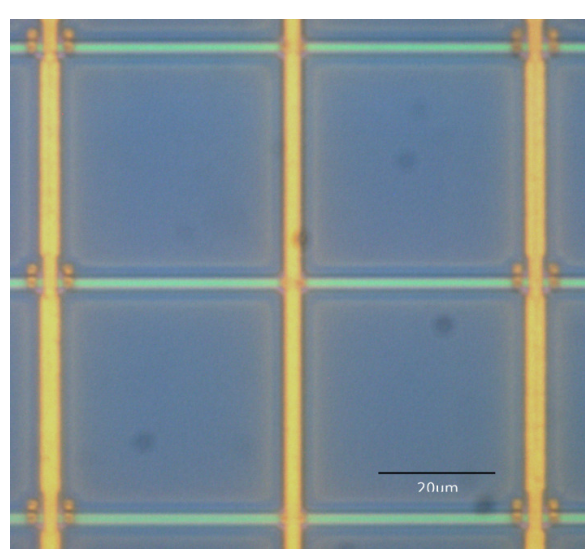

(c) SensL J-series 30035

Figure 2: Close-up pictures of the cells of the three tested SiPMs. The scale indicated by the black line in the images represents $20 \mu \mathrm{m}$.

avalanche current is eventually quenched. Furthermore, 69 all cells are connected to one common output. For a re- ${ }_{70}$ view of the history of SiPMs and their basic functionality, ${ }_{71}$ the reader is referred to [7] and references therein.

The three tested devices are

\section{- a NUV-HD SiPM from FBK,}

- a S13360-3050CS MPPC from Hamamatsu,

- and a MicroFJ-SMTPA-30035-E46 SiPM from SensL. 79

A picture of each SiPM is shown in Fig. 1. All three devices 81 are based on a $p$-on- $n$ structure, which means that the avalanche structure consists of a $p$-implant in an $n$-doped 82 substrate. In this configuration the electric field directs 83 electrons produced by blue photons just below the surface 84 into the high-field region, which is also why the sensitivity 85 of all three devices peaks at wavelengths in the blue or 86 near UV.

\section{1. $F B K N U V-H D$}

The FBK device is fabricated with NUV-HD technology [8]. The device investigated in this study has a custom geometry, which fits the requirements for the Cherenkov Telescope Array (CTA) [9] project. Unlike the other two devices, the NUV-HD does not have an epoxy, silicone resin, or similar protective coating. The dimensions of the FBK SiPM are $(6.8 \times 6.8) \mathrm{mm}^{2}$ with a micro-cell pitch of $30 \mu \mathrm{m}$. One SiPM has a total of 40,394 cells. The chip came glued onto a PCB carrier and is wire bonded. Fig. 2a shows a picture of four cells taken under a microscope. Clearly visible are the quench resistors (red) and the metal line that connects the output of all cells.

\subsection{Hamamatsu LCT5}

The SiPM from Hamamatsu is a S13360-3050CS MPPC [10]. It is fabricated using their latest technology, which is also called LCT5 because it is the fifth iteration of a low-cross-talk development. The dimensions of the tested device are $(3 \times 3) \mathrm{mm}^{2}$ with a cell pitch of $50 \mu \mathrm{m}$ (s. Fig. 
$2 \mathrm{~b}$ ) and a total of 3,600 cells. The device is mounted onto a ceramic chip carrier and coated with UV-transparent silicon resin. Electrical contacts between the chip and the pins of the carrier are made with wire bonds. Hamamatsu produces the same type of SiPM also with through-siliconvia (TSV) technology, which allows several chips to be packed into large matrices with minimal dead space.

\subsection{SensL J-Series}

The device from SensL is a pre-production J-Series SiPM [11]. The dimensions of the active area are $(3.07 \times$ $3.07) \mathrm{mm}^{2}$ and the cell pitch is about $41 \mu \mathrm{m}$ resulting in a total of 5,676 cells. The SiPM is embedded in a 4 -side tileable, chip scale package with TSV that is reflow soldered onto a PCB. The SiPM came surface mounted on an evaluation board (MicroF-SMTPA). A unique feature of SensL SiPMs is the presence of fast and slow readout terminals. The fast terminal capacitively couples directly to the cells, whereas the slow output is the conventional ${ }^{140}$ readout via the quench resistor. We used the signal from ${ }^{141}$ the slow terminal for our measurements.

\section{Photon detection efficiency}

The photon detection efficiency (PDE) quantifies the ${ }^{146}$ absolute efficiency of a photon detector to absorb a pho- ${ }^{147}$ ton and produce a measurable signal at its output. The PDE of SiPMs is determined by several factors of which the three most important are the geometrical efficiency, ${ }_{151}$ the quantum efficiency, and the probability to produce a Geiger breakdown, hereafter breakdown probability. The ${ }^{152}$ breakdown probability is also referred to as triggering ${ }_{154}$ probability.

We measure the PDE as a function of wavelength in three steps. In the first step, the PDE is measured at four ${ }_{156}$ wavelengths. In the second step, the relative spectral response is measured between $200 \mathrm{~nm}$ and $1000 \mathrm{~nm}$. In the $\mathrm{I}_{157}$ last step, the spectral response is scaled to match the four ${ }_{158}$ PDE points and thus arrive at the PDE for all wavelengths ${ }_{159}$ between $200 \mathrm{~nm}$ and $1000 \mathrm{~nm}$. In the following we walk in $_{160}$ detail through each of these steps. All PDE and spectral ${ }_{161}$ response measurements are carried out at room tempera- ${ }_{162}$ ture $\left(23^{\circ} \mathrm{C}-25^{\circ} \mathrm{C}\right)$.

\subsection{Concept of measuring the PDE}

The PDE at four different wavelengths is measured with ${ }^{164}$ the SiPM being biased above breakdown and illuminated ${ }^{165}$ with fast light flashes of known intensity, and from the $\mathrm{e}^{166}$ response of the SiPM the PDE is calculated. For the measurement we use the same procedure that is described $i^{167}$ $[12]$.

A pulsed LED flashes fast light pulses into an integrating ${ }^{169}$ sphere with two exit ports, which acts as an optical split- ${ }^{170}$ ter. The measurement of the splitting ratio is detailed in $^{171}$ section 3.3 A calibrated PiN diode is mounted to one exit port, and the SiPM under test is mounted to the other

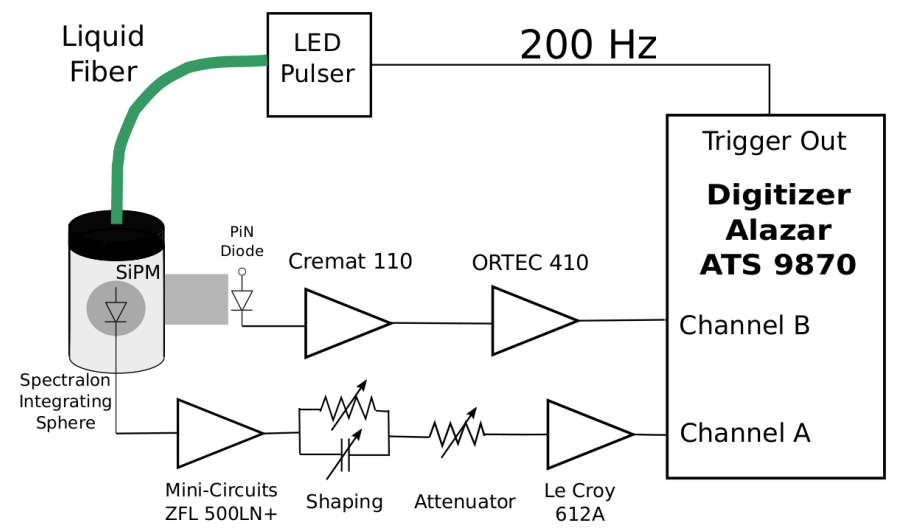

Figure 3: Sketch of the PDE setup.

port. The response of both sensors is recorded for each flash.

After 10,000 flashes, the average number of photons at the position of the SiPM is calculated from the average PiN-diode signal, the quantum efficiency of the PiN Diode, and the splitting ratio of the integrating sphere. The PDE of the SiPM then follows from the ratio of the average number of photons detected by the SiPM and the calculated average number of photons at the SiPM position.

The average number of photons and dark counts detected by the SiPM $\bar{N}_{\mathrm{Ph}+\text { DC }}$ in each flash is calculated under the assumption that the number of photons and dark counts in each flash follows a Poisson distribution. By counting the flashes $N_{0}$ for which the SiPM did not detect a photon, the average number of detected photons and dark counts is

$$
\bar{N}_{\mathrm{Ph}+\mathrm{DC}}=-\ln \left(\frac{N_{0}}{N_{\text {total }}}\right),
$$

where $N_{\text {total }}$ is the number flashes. The contribution from dark counts is determined by triggering the read out $N_{\text {total }}$ times without flashing the LED. As in the previous case, the number of times the SiPM did not record a signal $\left(N_{0}^{\mathrm{DC}}\right)$ is counted. The dark-count-subtracted average number of photons detected by the SiPM is then

$$
\bar{N}_{\mathrm{Ph}}=\ln \left(\frac{N_{0}^{\mathrm{DC}}}{N_{0}}\right) \text {. }
$$

The described procedure is commonly used to calculate the mean number of photons detected by SiPMs because it is immune to afterpulsing and optical crosstalk.

\subsection{PDE measurement setup}

The setup of our PDE measurement is sketched in Fig. 3. An LED pulses $20 \mathrm{~ns}-$ long flashes of light at $200 \mathrm{~Hz}$ into a UV-transparent liquid fiber that guides the light into a hollow cylinder made out of spectralon. ${ }^{1}$ The entry port

\footnotetext{
${ }^{1}$ The same integrating sphere that was also used in [12].
} 

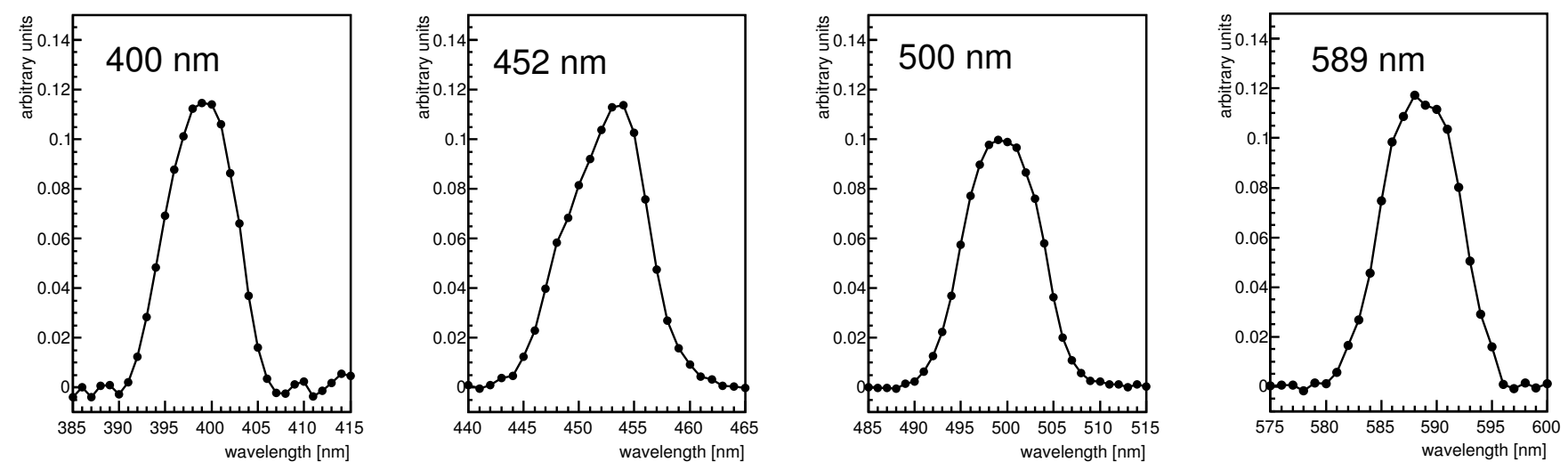

Figure 4: Spectra of the four LEDs after the light has passed through a narrow bandpass filter. The LEDs are operated in pulsed mode like in the PDE measurement.

and the two exit ports of the integrating sphere are all oriented perpendicular to each other. Attached to each exit port is an aluminum cylinder with the inside of the cylinder covered with black felt. Each cylinder is closed with a black plastic cap that has a hole in its center. A calibrated PiN diode is mounted to the cap with the larger hole ( $\sim 10 \mathrm{~mm}$ diameter), and the SiPM is mounted to the cap with the smaller hole $(\sim 1 \mathrm{~mm}$ diameter $)$.

Each SiPM is held in place with an adapter that is custom designed and 3D-printed for each device. The adapter ensures that only the active area of the SiPM is illuminated by the light that exits the integrating sphere through the end-cap of the aluminum cylinder. The diameter of the light beam is about $1 \mathrm{~mm}$. Four different LEDs fitted with narrow bandpass optical filters are used in the PDE measurement. The spectra of the four LEDs after the filter are shown in Fig. 4. The full width at half maximum (FWHM) of each spectrum is $\sim 10 \mathrm{~nm}$.

The PiN diode used in this study is a Hamamatsu S359008. The noise of the PiN-diode is minimized by reverse biasing the diode at $70 \mathrm{~V}$ thus decreasing the internal capacitance of the diode. The diode signal is first amplified ${ }_{211}$ with a Cremat 110 charge-sensitive preamplifier and then ${ }_{212}$ further amplified and shaped with an ORTEC Model $410_{213}$ linear amplifier. The best signal-to-noise ratio is achieved ${ }_{214}$ with $2 \mu$ s differentiating and integrating shaping time con-215 stants. The noise performance of the PiN-diode signal $_{216}$ chain is limited by the capacitance of the diode and the 217 intrinsic noise of the preamplifier and is about 300 equiva-218 lent noise charge (ENC). After amplification the signal is s $219_{2}$ recorded with an Alazar ATS 98708 bit, $1 \mathrm{GS} / \mathrm{s}$ digitizer. ${ }_{220}$

The SiPM signal is amplified with a Mini-Circuits 500-221 NL amplifier and then shaped with a simple variable par-222 allel $\mathrm{RC}$ circuit that differentiates the signal $(\mathrm{C})$ and pro-223 vides pole-zero cancellation $(\mathrm{R})$. After shaping, the typical 224 full width of the SiPM signal is less than $10 \mathrm{~ns}$. The sig-225 nal is further amplified with a LeCroy Model 612A ampli-226 fier before being digitized with the ATS 9870 digitizer. A227 switchable attenuator before the LeCroy amplifier is used 228

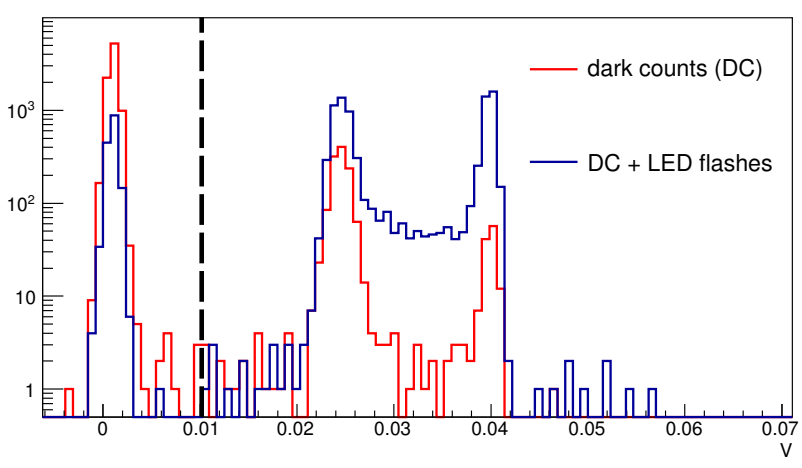

Figure 5: Pulse height distributions of Hamamatsu SiPM signals recorded in a PDE measurement. See text for details on the signal extraction. A total of 10,000 flashes contribute to each distribution. The blue distribution is from signals recorded when the SiPM is flashed with the LED. The red distribution is from signals recorded when the LED is not flashing. Events to the left side of the dashed vertical line can be identified as those in which the SiPM did not generate a signal.

to adjust the single photoelectron amplitude at the input of the digitizer to $\sim 30 \mathrm{mV}$.

The LED signal of the SiPM is extracted from the recorded trace by sliding a window of three samples (3ns) through the trace starting before the LED signal is expected in the trace and stopping $250 \mathrm{~ns}$ later. At each position the sum of the three samples is calculated, and at the end of the scan, the maximum sum is filled into a histogram. To extract the dark count rate, the procedure is repeated by starting $300 \mathrm{~ns}$ before the LED signal and sliding the three-sample window for another $250 \mathrm{~ns}$ through the trace stopping before the LED signal is expected in the trace. The maximum of the sliding window is again filled into a histogram. Fig. 5 shows the two resulting histograms for a typical measurement. Entries to the left of the dashed vertical line correspond to events during which the SiPM did not generate a signal within the $250 \mathrm{~ns}$. The integral of these events are $N_{0}^{\mathrm{DC}}$ (red histogram) and $N_{0}$ 
(blue histogram), respectively.

Note the good separation between the noise peak on the left and the first peak on the right side of the vertical line, which is necessary to keep the systematic uncertainties on the measured mean number of detected photons low. In all measurements the number of events in the minimum, where the dashed vertical line is placed, is $1 \%$ or less than the number of events in the maximum of the peak to the left. In that way the systematic uncertainty in the reconstructed mean number of photons is kept below $1 \%$.

The PiN diode signal is extracted by fitting a template pulse shape to the trace and recording the amplitude of the fitted pulse. The template pulse shape is averaged over 1000 pulses. The average number of photons at the PiNdiode position is calculated from the PiN-diode signals by taking the full LED spectrum and wavelength-dependent quantum efficiency $(\mathrm{QE})$ of the PiN diode into account.

\subsection{Calibration of the PDE setup}

Before a PDE value can be calculated, the $\mathrm{PiN}$ diode, 285 the integrating sphere, and the PiN diode signal chain need 286 to be calibrated. The Hamamatsu S3590-08 PiN diode has287 been calibrated by Hamamatsu, with a systematic uncer-288 tainty of $2-3 \%$ between $250 \mathrm{~nm}$ and $800 \mathrm{~nm}$ and up to $5 \%^{289}$ outside of that range [13].

\author{
290
}

For the measurement of the splitting ratio of the inte-291 grating sphere, S3590-08 PiN diodes are placed at the end 292 cap of each aluminum cylinder. An LED connected to a constant current source then shines into the entrance port ${ }^{293}$ of the integrating sphere. After one hour the LED has sta-294 bilized such that its intensity does not vary by more than 295 $0.1 \%$ over the course of one calibration measurement. 296

The currents of both PiN diodes are simultaneously297 recorded with two Keithley 6847 picoammeters. The photo current measured at the SiPM position (where the intensity is lowest) is at least 1000 times the PiN-diode dark $_{299}$ current. In a series of measurements the PiN diodes are $_{300}$ swapped.

The splitting ratio is first calculated by using the cur-302 rents that were measured with the same diode at the $\mathrm{two}_{303}$ exit ports. The ratio is then calculated a second time by b $_{30}$ using the currents that were measured with the two diodes ${ }_{305}$ simultaneously. In the final calculation, the currents $\operatorname{are}_{306}$ corrected for the small differences in the quantum efficien- 307 cies of the two PiN-diodes. All measurements of the split-308 ting ratio agree within $2 \%$. The ratio was, furthermore, 309 measured with all four LEDs used in the PDE measure- 310 ments and found to vary within $1 \%$.

The PiN-diode signal chain is calibrated in photoelec-312 trons by attaching a ${ }^{241} \mathrm{Am}$ source to the diode and record-313 ing the signals of $59.54 \mathrm{keV}$ gamma rays. Using a Fano 314 factor of $3.62 \mathrm{eV} /$ eh-pair it can be shown that the gamma315 rays produce on average 16448 eh-pairs in the diode [14]. A316 typical ${ }^{241} \mathrm{Am}$ spectrum recorded with our setup is shown ${ }_{317}$ in Fig. 6 together with pulse height distributions for each ${ }_{318}$ of the four LEDs.

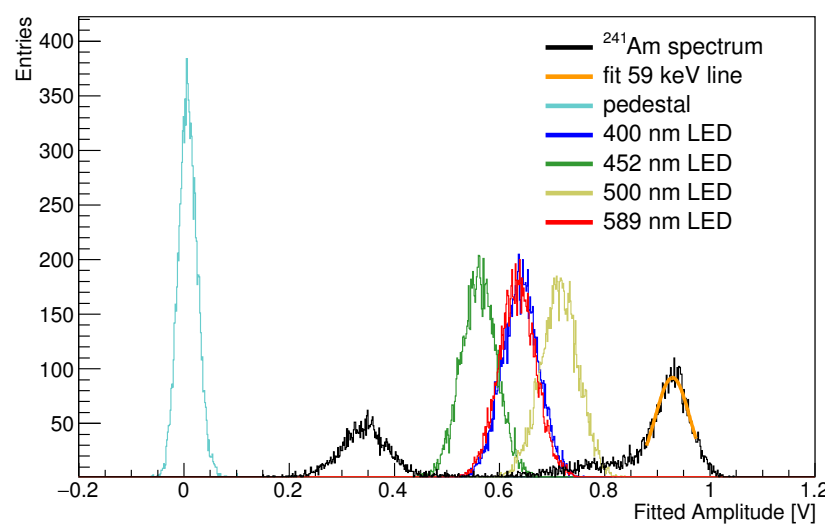

Figure 6: Pulse height distributions recorded with the calibrated PiN diode attached to the integrating sphere. Shown are distributions for all four LEDs, the ${ }^{241} \mathrm{Am}$ source, and the pedestal. The fit of the $59 \mathrm{keV}$ bin with a Gaussian function is also shown.

The linearity of the PiN-diode signal chain is better than $3 \%$ down to signal amplitudes that are $\sim 10 \%$ of an average $59 \mathrm{keV}$ signal.

We estimate that the relative systematic uncertainty of our PDE measurements is $5 \%$. The relative systematic uncertainty is dominated by systematic uncertainties of the PiN diode's QE (3\%), uncertainties in the ratio of the spectralon cylinder (1\%), and the signal extraction of the SiPM (1\%) and PiN diode $(3 \%)$.

\subsection{PDE measurements}

The PDE of all three devices is shown as a function of bias for all four wavelengths in Fig. 7. Each of the biasdependent PDE curves is well described by an exponential function of the form

$$
P D E(U)=P D E_{\max }\left[1-e^{-\left(U-U_{\mathrm{BD}}\right) / a}\right]
$$

with fit probabilities that are in all but one case better than $60 \%$. The good agreement indicates that the chosen analytical function is an appropriate empirical model of the data. The breakdown voltage $U_{\mathrm{BD}}$ is determined from the best fit of the $400 \mathrm{~nm}$ data and fixed in the fits of the data for the remaining wavelengths. The reasons for fixing the breakdown voltage are twofold. Firstly, the uncertainty of the best fit breakdown voltage is smallest in the fits of the $400 \mathrm{~nm}$ data, and secondly, the breakdown voltage does not depend on photon wavelength. We note that the breakdown voltages obtained here are in agreement with the dedicated breakdown-voltage measurements presented later.

The dashed vertical lines in Fig. 7 denote the bias at which each device reaches $90 \%$ of the maximum PDE at $400 \mathrm{~nm}$ as inferred from the fit of the data. For the remainder of this paper we refer to this bias voltage as the operating point of an SiPM and mark it accordingly in all figures with a downward pointing arrow. Note that the bias where the PDE reaches $90 \%$ of its maximum depends on wavelengths as will be discussed next. 


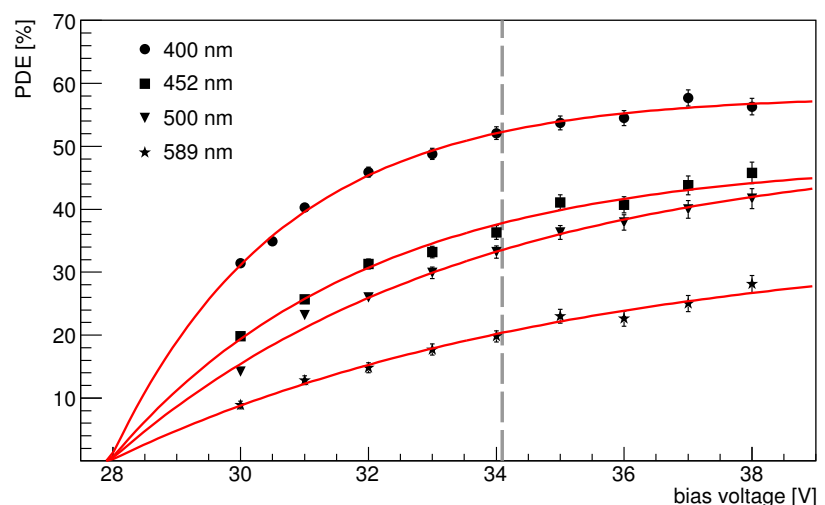

(a) FBK NUV-HD

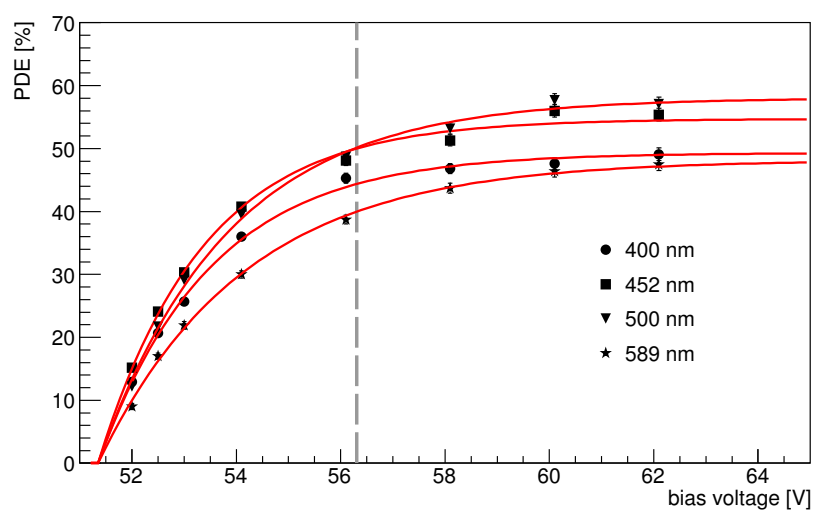

(b) Hamamatsu S13360-3050CS

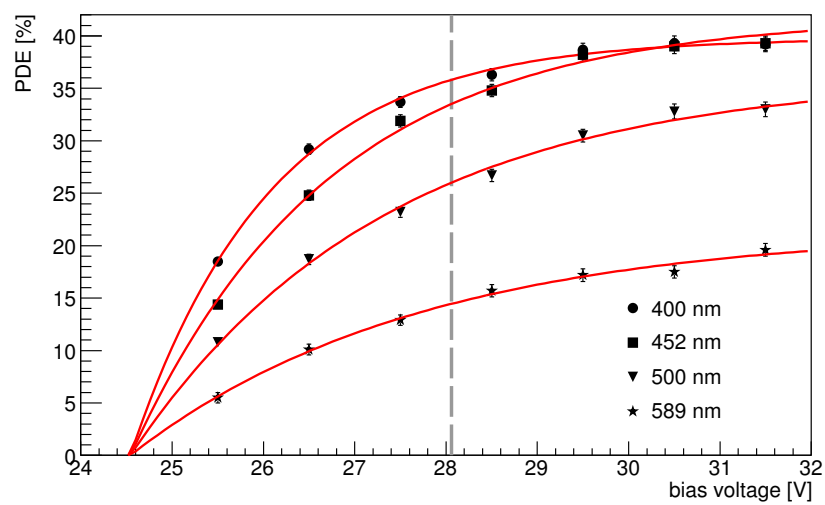

(c) SensL J-series 30035

Figure 7: PDE measured at four different wavelengths as a function of overvoltage.

The term in the square brackets in Equation 3 has to be interpreted as the breakdown probability, because the breakdown probability is the only contribution to the PDE that depends on bias, so long as the active volume of a cell is fully depleted (which can be safely assumed). After rewriting the exponent in units of relative overvoltage

$$
U_{\mathrm{rel}}=\frac{U-U_{\mathrm{BD}}}{U_{\mathrm{BD}}},
$$

which can in fact also be interpreted as the relative electric field strength above the critical electric field strength, the breakdown probability becomes

$$
P_{\mathrm{BD}}\left(U_{\text {rel }}\right)=1-e^{-U_{\text {rel }} / \alpha} .
$$

It is interesting to note that one parameter, $\alpha=a / U_{\mathrm{BD}}$, is sufficient to properly describe the electric field/bias dependence of the breakdown probability. The parameter $\alpha$ depends, of course, on the geometry of the avalanche region, where in the avalanche region a photon is absorbed, on the impact ionization factors of electrons and holes, and other factors and is thus device and wavelength specific. A small $\alpha$ value means that the breakdown probability rises quickly with bias as opposed to a slow rise if $\alpha$ is large. We discuss the interpretation of $\alpha$ in more detail in the following.

Fig. 8 shows the breakdown probability as a function of relative overvoltage / relative excess electric field for all three SiPMs and all four tested wavelengths. The corresponding values for $\alpha$ are listed in Table 1. All three devices have in common that $\alpha$ increases with increasing wavelength. This behaviour can be explained with the absorption length of photons, which increases with wavelength. For photons absorbed close to the surface of the SiPM (blue photons), it is the photoelectron that drifts into the avalanche region in $p$-on- $n$ devices. For photons absorbed below the avalanche region (redder photons), it is the hole that drifts upward into the avalanche region and initiates a breakdown. Because holes have always lower ionization factors than electrons, the breakdown probability for hole-dominated breakdowns is lower than for electron-dominated ones.

The ionization factors for electrons and holes grow rapidly with bias, therefore, the breakdown probability also increases until saturation is reached. Even though the ionization factor of holes increases faster than the one for electrons with bias it never becomes larger than the ionization factor of electrons. Thus the breakdown proba-

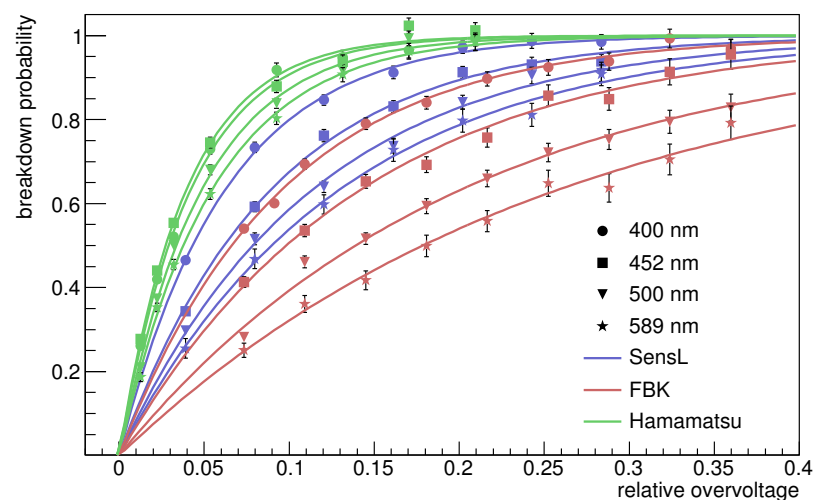

Figure 8: Breakdown probability as a function of relative overvoltage above breakdown for all three SiPMs and for all four wavelengths. The corresponding $\alpha$ values are listed in Table 1 . 
Table 1: $\alpha$ Values of the Fit Results in Fig. 8.

\begin{tabular}{c|c|c} 
Device & Wavelength & $\alpha$ \\
\hline \hline \multirow{2}{*}{ FBK } & $400 \mathrm{~nm}$ & $0.095 \pm 0.001$ \\
& $452 \mathrm{~nm}$ & $0.142 \pm 0.003$ \\
& $500 \mathrm{~nm}$ & $0.200 \pm 0.004$ \\
& $589 \mathrm{~nm}$ & $0.258 \pm 0.007$ \\
\hline Hamamatsu & $400 \mathrm{~nm}$ & $0.0420 \pm 0.0005$ \\
& $452 \mathrm{~nm}$ & $0.0395 \pm 0.0006$ \\
& $500 \mathrm{~nm}$ & $0.0485 \pm 0.0007$ \\
& $589 \mathrm{~nm}$ & $0.0546 \pm 0.0010$ \\
\hline \multirow{2}{*}{ SensL } & $400 \mathrm{~nm}$ & $0.062 \pm 0.001$ \\
& $452 \mathrm{~nm}$ & $0.089 \pm 0.002$ \\
& $500 \mathrm{~nm}$ & $0.113 \pm 0.002$ \\
& $589 \mathrm{~nm}$ & $0.129 \pm 0.004$
\end{tabular}

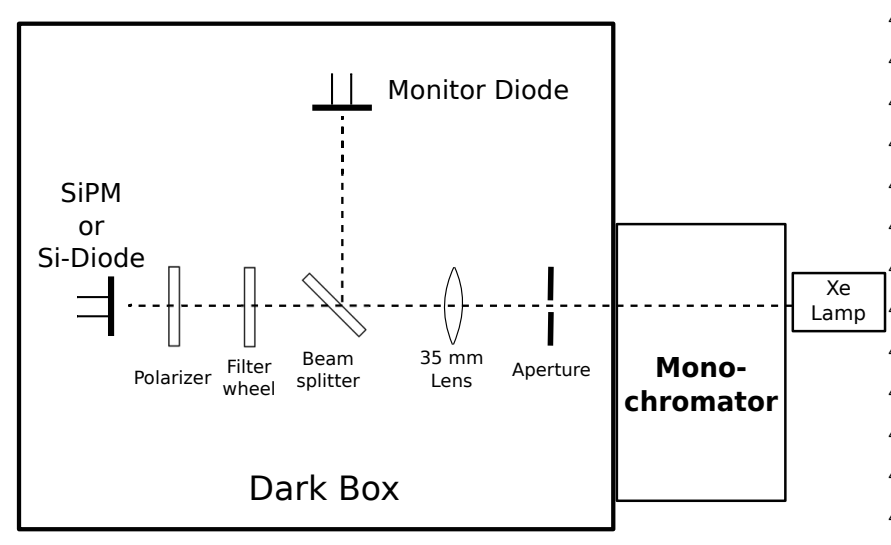

Figure 9: Sketch of the spectral response setup.

bility for longer wavelengths is always less than for shorter ${ }_{419}^{418}$ wavelengths and saturation is reached at a higher bias. ${ }^{419}$

The Hamamatsu SiPM has the lowest $\alpha$ of the three ${ }^{420}$ devices at all wavelengths, while the FBK device features ${ }^{42}$ the largest $\alpha$ values. These differences can be qualitatively ${ }^{422}$ attributed to differences in the location of the avalanche ${ }^{423}$ region (how close it is to the surface), spatial extent of the avalanche region, the geometry of the avalanche region, ${ }_{426}^{425}$ and variations of it when the bias is being changed.

It is evident that all three devices can be operated at ${ }^{427}$ a breakdown probability of $90 \%$ or more - at least in the ${ }^{428}$ blue. This is a significant improvement compared to $\mathrm{a}^{429}$ few years ago when most devices could only operate at a maximum overvoltage of 5\%-10\%, and, therefore, yielded ${ }^{431}$ much lower breakdown probabilities [6].

\subsection{Concept of the spectral response measurement}

For the spectral response measurement, we use the setup 436 that is sketched in Fig. 9. The SiPM is biased at the 437 voltage that yields a $90 \%$ breakdown probability at $400 \mathrm{~nm}_{438}$ as defined in the previous section. The SiPM is measured $d_{439}$ first and then replaced with the reference detector instead of measuring both sensors simultaneously like in the PDE measurement. Doing the spectral response measurement in this way eliminates optical elements that split the light between the two sensors and therefore would have to be calibrated. A main source of systematic uncertainties is thus eliminated.

Any variability of the light source is monitored and recorded with a permanently installed PiN diode. Further corrections that are applied in the data analysis are a) subtraction of dark currents of all sensors and b) subtraction of stray light transmitted through the monochromator, which affects measurements mainly below $350 \mathrm{~nm}$.

The intensity of the light source is adjusted throughout a measurement by controlling the slits of the monochromator such that the SiPM current is within 50 to 75 times the dark current of the SiPM. Keeping the current of the SiPM quasi-constant guarantees that the fraction of SiPM cells that are in recovery remains about the same, and thus the geometrical efficiency of the SiPM also remains constant. The current limits are such that only a small fraction of the cells of an $\mathrm{SiPM}(<1 \%)$ are always in recovery and, therefore, saturation effects of the SiPM are avoided. The light spot at the position of the SiPM is larger than the sensor itself. Each spectral response measurement is crosschecked by increasing the current limits to be between 100 and 150 times the dark current and making sure that the residuals between the two measurements remain less than $2 \%$.

The spectral response measurement is a relative one and is converted into an absolute PDE measurement by fitting it to the PDE measurements presented earlier. Corrections for optical crosstalk and afterpulsing, therefore, do not have to be applied to the spectral response measurements.

\subsection{Setup of the spectral response measurement}

The light source in the spectral response measurement is a $300 \mathrm{~W}$ UV-enhanced Xenon arc lamp (PE300BUV from Cermax). The light of the lamp is air-coupled into a Czerny-Turner single-grating monochromator Digikröm DK $2401 / 4 \lambda$ from Spectral Products. The grating of the monochromator that is used for all measurements has 1200 grooves per millimeter and a $300 \mathrm{~nm}$ blaze wavelength. The output of the monochromator is coupled into a dark box where the light beam is further conditioned before it illuminates the monitoring diode and the SiPM or reference sensor.

Inside the dark box the light first passes an adjustable aperture followed by a lens with a focal length of $35 \mathrm{~mm}$. The beam is then split by a polka dot beamsplitter. The reflected part of the beam illuminates the monitoring diode - an unbiased Hamamatsu S3590-08 PiN diode. The size of the beam spot matches the size of the monitoring diode.

The transmitted part of the beam passes through an optical long-pass filter that is mounted onto a filter wheel, followed by an optional broadband polarizer (UBB01A from 
Moxtek) before the beam illuminates either the SiPM or the reference sensor. The beam spot is larger then the size of the reference sensor or the SiPM. The reference sensor is a UV-enhanced, Si-diode from Hamamatsu (type S12271010BQ, calibrated by Hamamatsu). All optical elements are UV transparent down to $200 \mathrm{~nm}$.

A total of three long-pass filters with cut-off wavelengths at $280 \mathrm{~nm}, 400 \mathrm{~nm}$, and $750 \mathrm{~nm}$ are mounted into a computer-controlled filter wheel. The $280 \mathrm{~nm}$ filter is used to quantify stray light with wavelengths above the cut-off wavelength that gets transmitted through the monochromator and affects measurements below $270 \mathrm{~nm}$. The $400 \mathrm{~nm}$ filter is used to quantify the stray-light component that affects measurements between $270 \mathrm{~nm}$ and $350 \mathrm{~nm}$. The $400 \mathrm{~nm}$ filter is also used to suppress higherorder diffraction above $430 \mathrm{~nm}$. The $700 \mathrm{~nm}$ filter suppresses higher-order diffraction above $770 \mathrm{~nm}$.

The current of the monitoring diode is recorded with a Keithley 6845 picoammeter, and the currents of the reference sensor and the SiPM are measured with a Keithley ${ }_{496}$ 6847 picoammeter. The readings of both instruments are transfered via serial link to a computer, which also controls ${ }_{498}^{497}$ the monochromator and the filter wheel.

For the spectral response measurement, the $\mathrm{SiPM}$ is $_{500}^{499}$ fixed on a rotary mount that allows making spectral response measurements as a function of the angle of incidence between 0 degrees (normal incidence) and 90 de- $^{-501}$ grees. The SiPM is biased with the internal voltage source502 of the Keithley 6847 picoammeter.

In the measurement the monochromator output is504 changed between $200 \mathrm{~nm}$ and $1000 \mathrm{~nm}$ and for each wave-505 length the exit and entrance slits of the monochromatorso6 is adjusted to keep the SiPM current within the previ-507 ously discussed limit of 50-75 times the SiPM's dark cur-508 rent. The long-pass filters are inserted at the above men-509 tioned wavelengths. The SiPM is then swapped out with510 the calibrated Si-diode, and the photocurrent of the diodesi1 is recorded at the same wavelengths and with the same512 monochromator slit settings used in the SiPM measure-513 ment.

The spectral response $S$ at a given wavelength is calcu-515 lated as

$$
S=\frac{I_{\mathrm{SiPM}}}{I_{\mathrm{Si-Diode}}} \cdot \frac{I_{\text {Mon. Si-Diode }}}{I_{\text {Mon. SiPM }}} \cdot Q E_{\text {Si-Diode }},
$$

where $I_{\mathrm{SiPM}}$ and $I_{\mathrm{Si} \text {-Diode }}$ are the dark and the stray-light520 corrected currents of the SiPM and the calibrated Si-diode,521 respectively. The factor in the middle is the ratio of the 522 dark-current-subtracted currents of the monitoring diode 223 that corrects for fluctuations of the Xe lamp. The last ${ }_{524}$ factor $Q E_{\text {Si-Diode }}$ is the quantum efficiency of the reference525 sensor.

The systematic uncertainties between $300 \mathrm{~nm}$ and ${ }_{527}$ $800 \mathrm{~nm}$ are dominated by uncertainties in the wavelength-528 dependent response of the calibrated Si-diode $(\sim 3 \%)$ and 529 variations in the $\mathrm{SiPM}$ photocurrent that cause the frac-530 tion of recovering SiPM cells to vary accordingly $(\sim 1 \%) .531$

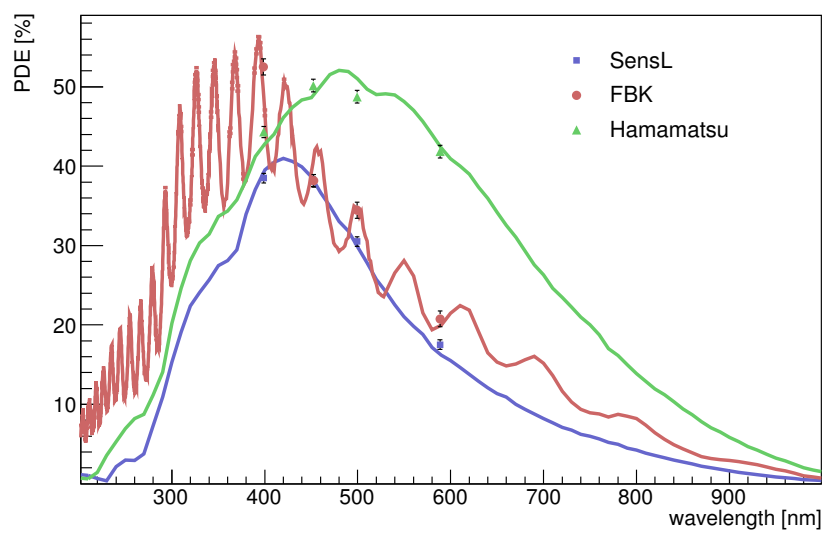

Figure 10: PDE vs. wavelength for all three devices between $200 \mathrm{~nm}$ and $1000 \mathrm{~nm}$. The bias voltage for each device results in a $90 \%$ breakdown probability at $400 \mathrm{~nm}$, the operating point of each SiPM.

Below $300 \mathrm{~nm}$ the systematic uncertainties are dominated by residuals in the stray-light correction when the PDE of the SiPM drops below $10 \%$. They reach $100 \%$ when the PDE of the SiPM drops below a couple of percent. Above $800 \mathrm{~nm}$ the uncertainties are dominated by the uncertainty in the $\mathrm{QE}$ of the reference sensor, which is $\sim 4 \%$.

\subsection{Wavelength dependent PDE}

The spectral response measurement is a relative one and converted into an absolute PDE measurement by fitting it to the previously discussed PDE measurements at four wavelengths. The fit is done by invoking a scaling factor that minimizes $\chi^{2}$ between the four PDE points and the spectral response measurements. In the fit it is taken into account that the spectral response of the SiPM varies across the spectra of the LEDs that have been used in the PDE measurements. In order to find the correct wavelength that corresponds to the measured PDE, an LED spectrum is weighted with the spectral response of the $\mathrm{SiPM}$, and the mean wavelength of the weighted spectrum is used as the wavelength of the PDE measurement. The correction, however, is small, and the shift with respect to the mean LED wavelength is $<1 \mathrm{~nm}$. Afterpulsing and optical crosstalk do not affect the outcome of the scaling because both result in a wavelength-independent factor that gets marginalized in the fit.

The spectral response measurements scaled to absolute PDE are shown in Fig. 10. Also shown are the four PDE measurements for each device to which the spectral response measurements have been scaled.

The FBK device has the highest peak PDE of the three tested SiPMs with $56 \%$ at $395 \mathrm{~nm}$, even though it has the smallest pitch between cells. The oscillations in the PDE are due to interference caused by the thin passivation layer and the lack of a coating on top of the device like in the other two devices. In a previous study we tested an NUVHD device with coating that shows a comparable PDE down to $300 \mathrm{~nm}$. Below $300 \mathrm{~nm}$ FBK device presented here 
has a better efficiency because it is not coated with silicon resin. The full width at half maximum (FWHM) of the FBK PDE extends from $280 \mathrm{~nm}$ to $560 \mathrm{~nm}$. The Hamamatsu device has a peak PDE of $52 \%$ at $455 \mathrm{~nm}$ and a FWHM of the PDE response that extends from $310 \mathrm{~nm}$ to $700 \mathrm{~nm}$, which is significantly more red sensitive than the FBK SiPM. The SensL device has a peak PDE of $41 \%$ at $420 \mathrm{~nm}$ and a FWHM of the PDE response from $310 \mathrm{~nm}$ and $560 \mathrm{~nm}$, which is similar to the response of the FBK SiPM.

Compared to similar SiPMs from only a few years ago [6], all three devices are testaments to the major improvements that have been made in increasing the PDE and shifting the response of SiPMs to shorter wavelengths.

\subsection{Dependence of SiPM response on angle of incidence}

The dependence of the PDE on the angle of incidence was tested for light polarized in the plane of incidence (parallel polarization) and perpendicular to the plane of incidence for angles incidence angles of $20^{\circ}, 40^{\circ}, 50^{\circ}, 60^{\circ}$, and $70^{\circ}$. For this measurement a broadband polarizer UBB01A from Moxtek was inserted after the beam splitter. Fig. 11 shows the response of the three SiPMs relative to normal incidence for polarization perpendicular to the plane of incidence and in Fig. 12 for light polarized parallel to the plane of incidence. The measurements are corrected for the change in the projected area of the light beam onto the SiPM with different angle of incidence. We estimate a maximum uncertainty on the angle of incidence of $2^{\circ}$, which translates into a maximum systematic uncertainty of $10 \%$ on the measurements done at $70^{\circ}$ and less at smaller angles.

The response to different angles of incidence depends to a large fraction on the coating of the chip and also how the chip is packaged. In order to reduce effects from stray light that reflects off the chip carrier into the edges of the chip or light that directly enters through the edges of the chip under larger angles, the boundaries of the Hamamatsu and the SensL SiPM were covered with thin copper tape. Unfortunately, the FBK SiPM could not be taped because the chip is not protected, thus edge effects are included in the measurement.

The response of all devices is relatively insensitive up to angles of $60^{\circ}$, when the response is still about $80 \%$ and better than $90 \%$ for perpendicular and parallel polarized light, respectively. At larger angles the sensitivity starts to quickly drop. Note that there is a steep increase in sensitivity of the SensL device to parallel polarized light between $300 \mathrm{~nm}$ and $400 \mathrm{~nm}$ for larger angles of incidence.

\section{IV curves}

For the measurement of the electrical characteristics, these SiPMs are placed in a thermal chamber, and their perfor-589 mance is measured between $-40^{\circ} \mathrm{C}$ and $40^{\circ} \mathrm{C}$ in steps of 590 $20^{\circ} \mathrm{C}$. Fig. 13 shows a sketch of the setup.

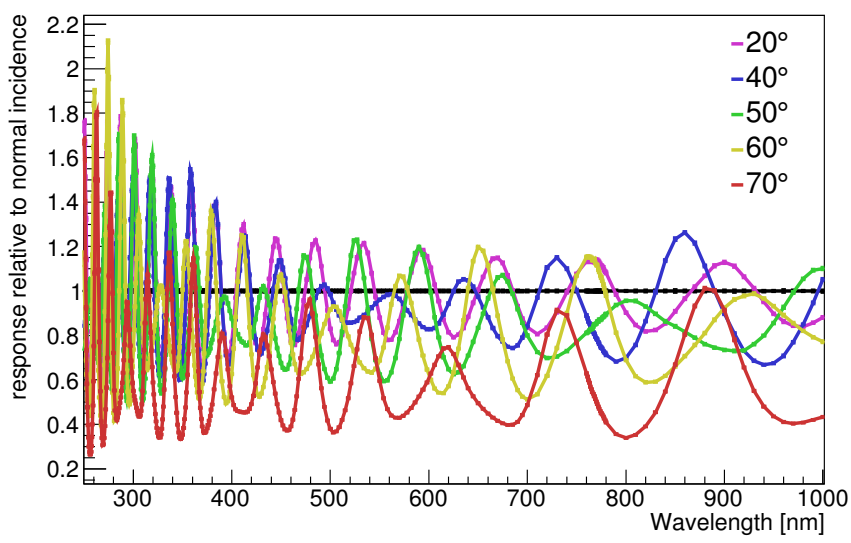

(a) FBK NUV-HD

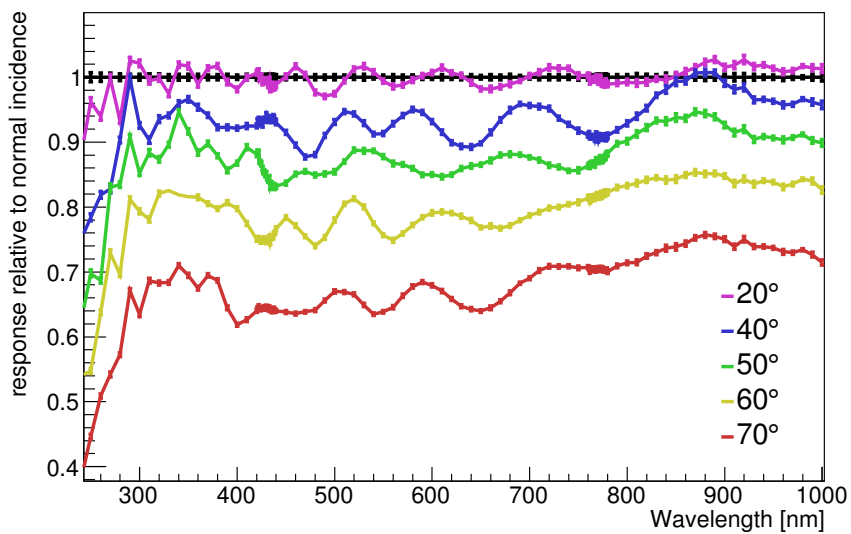

(b) Hamamatsu S13360-3050CS

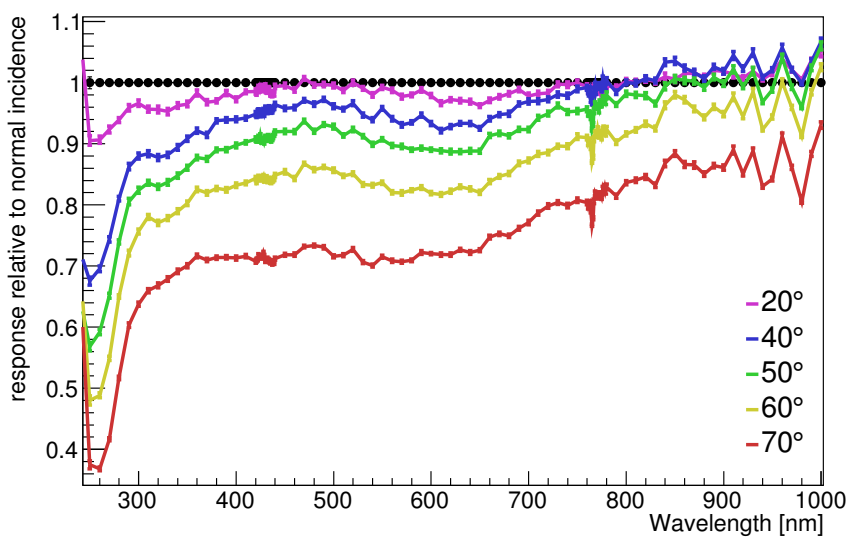

(c) SensL J-series 30035

Figure 11: Response as a function of angle of incidence relative to normal incidence with light polarized perpendicular to the plane of incidence.
.

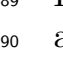
.
In this section the $I V$-curve measurements are discussed. For each measurement, each SiPM is connected to a Keithley 6847 picoammeter that biases the SiPM and records the current. The measurements are done in DC mode as opposed to a pulsed mode, which is acceptable given the small amount of power dissipated by the $\mathrm{SiPM}(<20 \mathrm{~mW}$ when biased in the forward direction and 


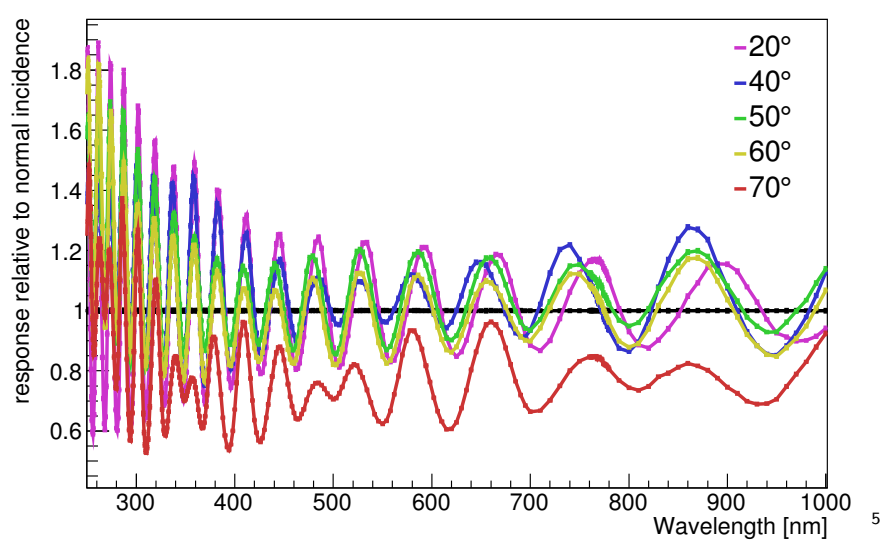

(a) FBK NUV-HD

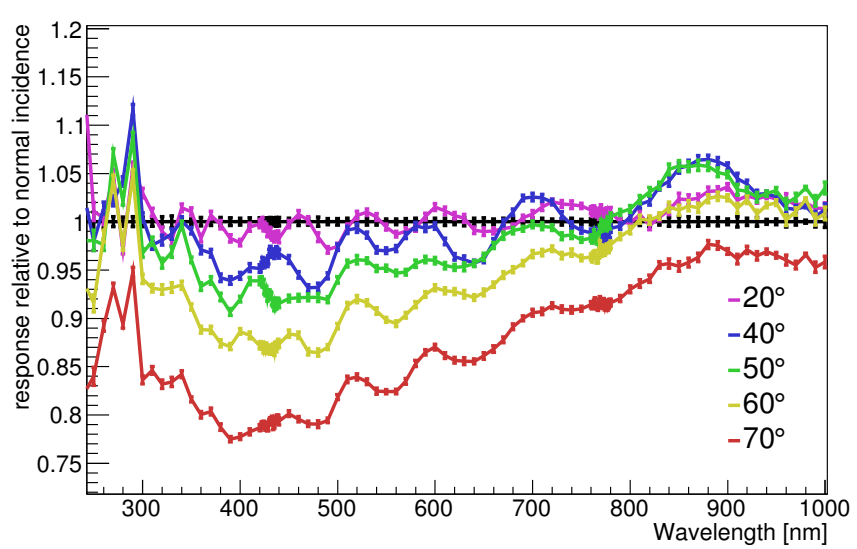

(b) Hamamatsu S13360-3050CS

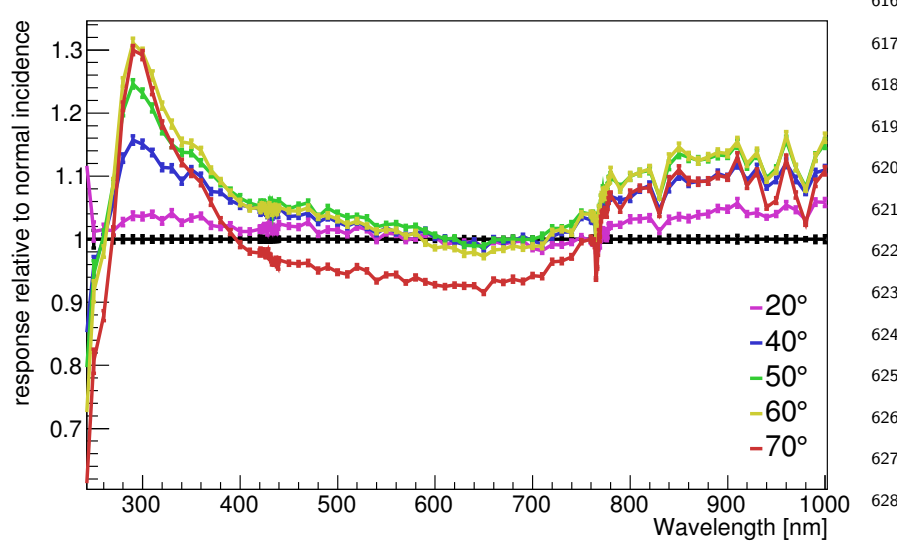

(c) SensL J-series 30035

Figure 12: Response as a function of angle of incidence relative to normal incidence with light polarized parallel to the plane of inci- ${ }^{630}$ dence. $<1 \mathrm{nW}$ when biased in reverse). From the $I V$-curves the ${ }_{634}$ average value of the quench resistor and the breakdown ${ }_{635}$ voltage are derived.

\subsection{Quench resistor values}

The quench resistor values are derived from the linear part of the forward biased $I V$ curves (see Fig. 14), i.e. in 640

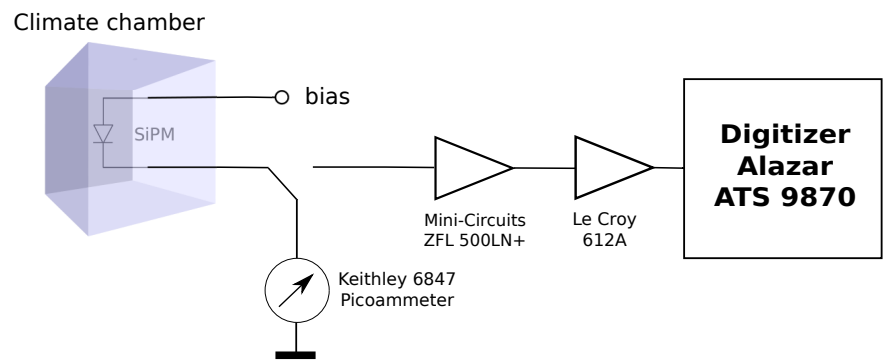

Figure 13: Sketch of the basic measurement setup

the regime where the resistance of the $p n$-junction of a cell becomes negligible, and the total resistance is dominated by that of the quench resistor.

The inverse of the slope of the $I V$ curve yields the resistance of all quench resistors of the SiPM connected in parallel. Multiplying the total parallel resistance with the number of cells of an SiPM thus gives the average value of a quench resistor, which is shown in Fig. 15 as a function of temperature for all three SiPMs.

The figure also gives the temperature coefficients of the quench resistors, which are determined by fitting a linear function to the data points, which is a good approximation for the Hamamatsu and SensL data. For the FBK SiPM, the quench resistor values fluctuate significantly. In particular the value at $40^{\circ} \mathrm{C}$ is higher than one would expect by extrapolating the quench resistor values from lower temperatures. We can not exclude that a contamination of the uncoated device during handling or residual humidity is responsible for these effects.

The quench resistors of the Hamamatsu device have the smallest relative dependence on temperature with $2 \cdot 10^{-3}$, followed by $3 \cdot 10^{-3}$ for the SensL device, and $5 \cdot 10^{-3}$ for the FBK device. The temperature coefficient and the absolute value of the quench resistor determine the maximum temperature and bias at which a device can be operated before a breakdown cannot be reliably quenched anymore. It, furthermore, determines how the recovery time of a cell changes with temperature. The temperature coefficients of all three SiPMs, however, are too small to have any practical impact on the maximum operating temperature or cell recovery times.

\subsection{Breakdown voltages}

The second characteristic derived from the $I V$-curves is the breakdown voltage. We took a close look at three different proposed methods $[15,16,17]$ to extract the breakdown voltage, and we compare them with the classical method that uses gain vs. bias measurements. Based on our findings we propose yet another method that is based on $[15,16]$ and yields breakdown voltages within $\pm 2 \cdot 10^{-3}$ of the true value.

It has been noted, based on empirical evidence, that the $I V$ curve of single SiPM cells (also called SPADs) can be described by a parabola above breakdown [18]. Here 


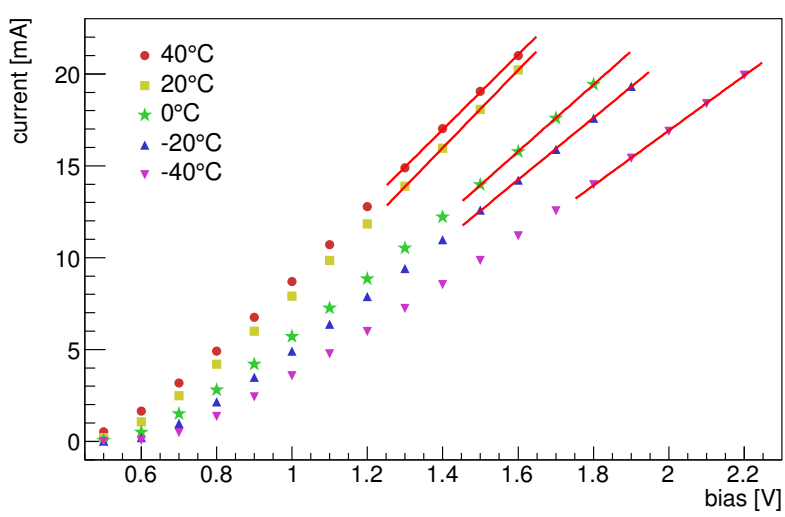

(a) FBK NUV-HD

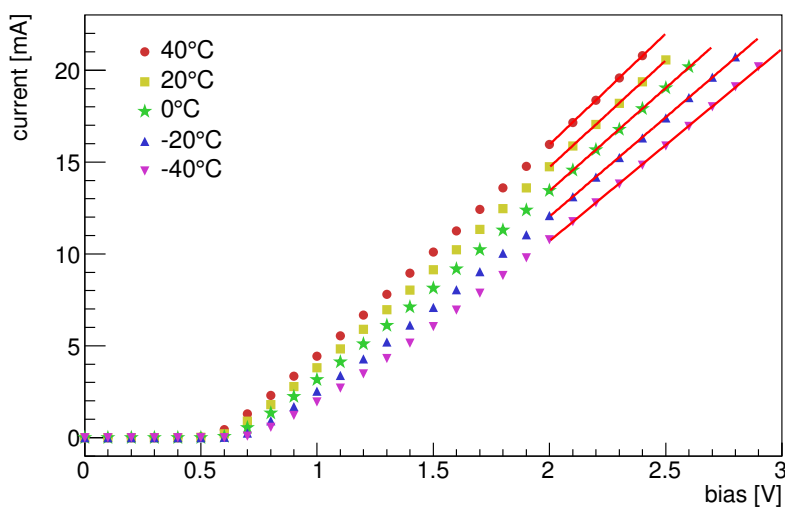

(b) Hamamatsu S13360-3050CS

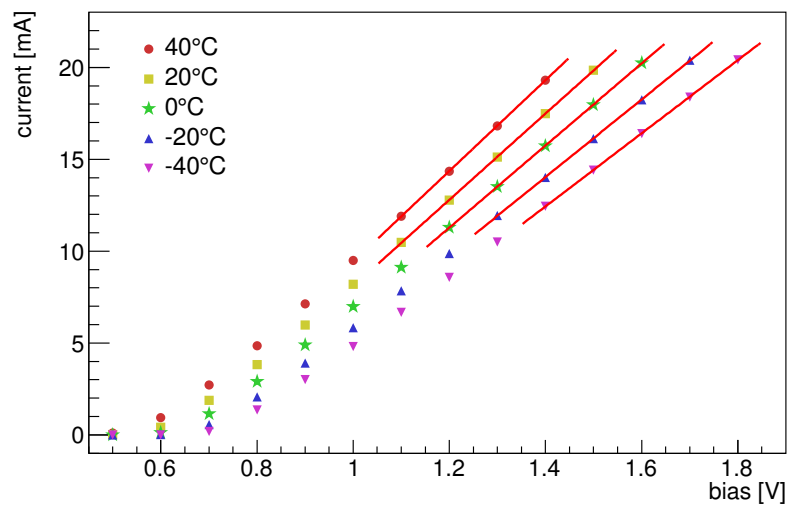

(c) SensL J-series 30035

Figure 14: $I V$ curves of all $\mathrm{SiPMs}$ biased in the forward direction at five different temperatures. The solid lines are fits with linear functions, which are used to derive the average quench resistor value. The measured quench resistor values are shown in Fig. 15. See text for further details.

we give a physical explanation why a parabola is in fact ${ }^{647}$ expected for the $I V$ curve just above breakdown.

Biased just above breakdown, the current is proportional to the product of gain $G=C \cdot \Delta U=C \cdot U_{\mathrm{BD}} \cdot U_{\mathrm{rel}}$ and breakdown probability $1-\exp \left(-U_{\text {rel }} / \alpha\right)$, where $C$ is the

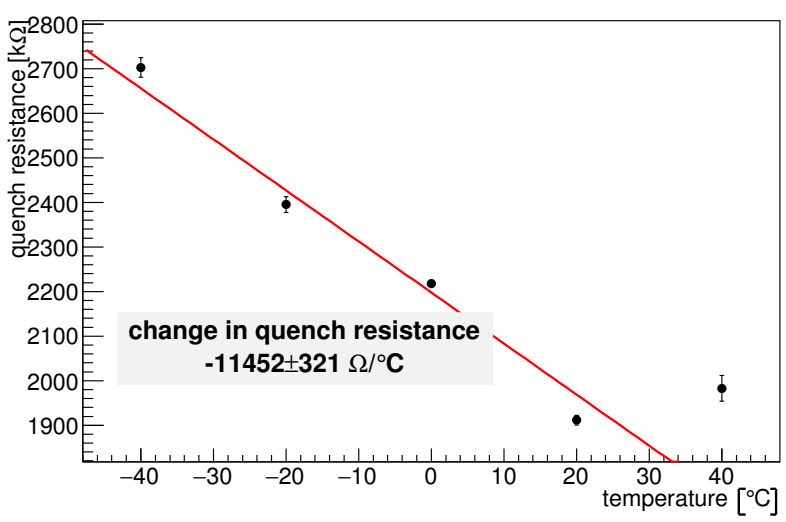

(a) FBK NUV-HD

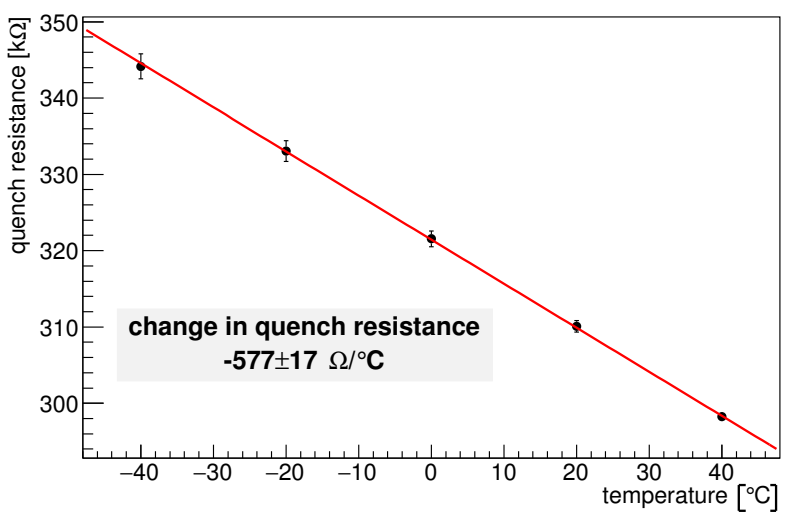

(b) Hamamatsu S13360-3050CS

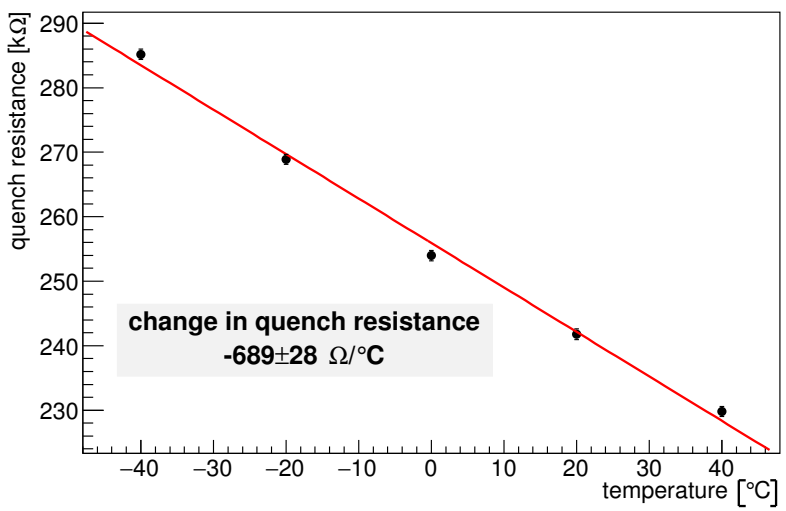

(c) SensL J-series 30035

Figure 15: Average quench-resistor values for all three SiPMs at five different temperatures. The change in resistance with temperature shown in each figure is determined from a fit of the data points with a linear function.

effective capacitance of one $\mathrm{SiPM}$ cell $^{2}$ and $\Delta U=U-U_{\mathrm{BD}}$. The proportionality constant is the sum of the dark current $I_{\mathrm{DC}}$ and the current due to external light sources

\footnotetext{
${ }^{2}$ The cell capacitance is determined from gain vs. bias measure-
} ments and is discussed later. 
$I_{\text {ext }}$ multiplied by the optical crosstalk probability $P_{\mathrm{OC} 686}$ and afterpulsing probability $P_{\mathrm{AP}}$. The total current above 687 breakdown is then

$$
\begin{aligned}
I\left(U_{\mathrm{rel}}\right)= & {\left[I_{\mathrm{DC}}\left(U_{\mathrm{rel}}\right)+I_{\mathrm{ext}}\right] } \\
& \cdot\left[1+P_{\mathrm{OC}}\left(U_{\mathrm{rel}}\right)+P_{\mathrm{AP}}\left(U_{\mathrm{rel}}\right)\right] \\
& \cdot C \cdot U_{\mathrm{BD}} \cdot U_{\mathrm{rel}} \cdot\left[1-e^{\left(-U_{\mathrm{rel}} / \alpha\right)}\right] .
\end{aligned}
$$

The dark current changes much less with bias than the ${ }^{694}$ breakdown probability, and the gain and can thus be as- ${ }^{695}$ sumed constant if only a small range around the break- ${ }^{696}$ down voltage is considered. The impact of a varying dark ${ }^{697}$ current is further suppressed by illuminating the $\mathrm{SiPM}^{698}$ with an external light source that produces a current that ${ }^{699}$ is ten times or more than the SiPM dark current. ${ }^{3}$ In fact, ${ }^{700}$ for this method to also work at low temperatures when the ${ }^{701}$ dark current becomes too low to provide a large enough ${ }^{702}$ primary signal, an external light source is needed.

Optical crosstalk and afterpulsing are only a few per- ${ }^{704}$ cent around the breakdown voltage and can, therefore, be ${ }^{705}$ neglected. With these simplifications the total current be- ${ }^{706}$ comes

$$
\begin{aligned}
I\left(U_{\mathrm{rel}}\right) \approx & {\left[I_{\mathrm{DC}}+I_{\mathrm{ext}}\right] \cdot C \cdot U_{\mathrm{BD}} } \\
& \cdot U_{\mathrm{rel}} \cdot\left[1-e^{\left(-U_{\mathrm{rel}} / \alpha\right)}\right] .
\end{aligned}
$$

Doing a series expansion of the exponential function $t^{712}$ second order in $U_{\text {rel }} / \alpha$ gives

$$
\begin{aligned}
I\left(U_{\mathrm{rel}}\right) \approx & {\left[I_{\mathrm{DC}}+I_{\mathrm{ext}}\right] \cdot C \cdot U_{\mathrm{BD}} } \\
& \cdot\left[U_{\mathrm{rel}}^{2} / \alpha+U_{\mathrm{rel}}^{3} / 2 \alpha^{2}+\ldots\right] .
\end{aligned}
$$

Thus in leading order the current above breakdown is in- ${ }^{718}$ deed proportional to $\Delta U^{2}$ as long as $U_{\text {rel }} / \alpha<1$, which is ${ }^{719}$ the case for overvoltages that are less than $5 \%-10 \%\left(\mathrm{see}^{720}\right.$ Table 1).

To obtain the breakdown voltage from an $I V$ curve, ${ }^{722}$ [16] proposes using the voltage where $(\mathrm{d} I / \mathrm{d} U) / I$ is ${ }^{723}$ maximal, whereas [15] proposes using the maximum of ${ }^{724}$ $\mathrm{d} \ln (I(U)) / \mathrm{d} U$. Both methods are equivalent because if ${ }^{725}$ applied to Eqn. 8 both yield

$$
\frac{\mathrm{d} I / \mathrm{d} U}{I}=\frac{\mathrm{d} \ln (I(U))}{\mathrm{d} U}=\frac{2+f(y)}{U-U_{\mathrm{BD}}} .
$$

The function $f(y)=(y+1-\exp (y)) /(\exp (y)-1)$, with $_{73}{ }^{730}$ $y=U_{\text {rel }} / \alpha$, is about -0.2 for values of $y$ that are typical ${ }_{732}$ for the tested devices.

We verified that processing our $I V$ measurements in both ways does indeed yield identical results. Fig. $16_{735}$ shows the outcome when they are processed according to $\mathrm{O}_{736}$ $\mathrm{d} \ln (I(U)) / \mathrm{d} U$. In all of these measurements the $\mathrm{SiPMs}_{737}$ were illuminated with a dimmed $400 \mathrm{~nm}$ LED.

${ }^{3}$ An external light source that produces a current 100 times the ${ }^{739}$ dark current will not affect the response of the SiPM (see spectral ${ }^{740}$ response measurement section).
The peak positions shown in Fig. 16 are systematically above the breakdown voltage derived from gain vs. bias measurements by about $0.7 \%$, which is not acceptable in some applications. In an effort to obtain a better estimate of the breakdown voltage, we fit each curve in Fig. 16 with Eqn. 10. The results of the fits are shown in Fig. 16 on top of the data.

The breakdown voltages extracted from the fit are shown together with those from the gain measurements in Fig. 17. Differences between the fitting method and the gain method are less than $\pm 0.2 \%$, which is significantly better than the $0.7 \%$ offset observed in the peak-derivative method. Some of the remaining offset can be explained with systematic uncertainties in the calibration of the signal chain that is used in the gain vs. bias measurements.

An obvious outlier is the result obtained for the Hamamatsu SiPM where all breakdown voltages derived from the $I V$ curve have a relative offset of $0.4 \%$ from the gain vs. bias derived breakdown voltages, which is too large an offset to be explained by calibration uncertainties. The measurement of the breakdown voltage done by Hamamatsu agrees with the one from our gain vs. bias measurement.

We cannot exclude with certainty that variations of the cell capacitance with bias might be a possible cause for the discrepancy in the breakdown voltage measurements. But we note that the gain vs. bias curves in Figure 20 are linear down to 1 Volt overvoltage. Thus any significant change in the cell capacitance must happen around the breakdown voltage and thus invalidate the model of the $I V$ curve (Equation 7) and the gain method, which both assume a constant cell capacitance.

An additional benefit of the fit is that it also extracts values for $\alpha$. For all three devices the fit produces $\alpha$-values at room temperature that are consistent with those listed in Table 1. The data seem to indicate a weak increase of $\alpha$ with temperature but the uncertainties are too large to make a more quantitative statement.

The last method we investigated to extract the breakdown voltage from the $I V$ curve is to use the maximum of the second derivative of the logarithm of the current [17]. The estimated breakdown voltages are shown in Fig. 17 as open squares and yield a similarly good estimate of the breakdown voltage as our fitting method. For the Hamamatsu SiPM the position of the maximum of the second derivative gives slightly better results, but it is still offset from the true breakdown voltage.

The breakdown voltages in Fig. 17 change proportionally with temperature for all three devices. The temperature coefficients of the breakdown voltage are given in the same figure. The relative change in breakdown voltage with temperature is about the same for all three devices, namely $10^{-3}$ per one degree Celsius.

\subsubsection{IV curve simulations in the breakdown region}

We simulated $I V$ curves for two reasons. First we want to explain why the position of the maximum in the deriva- 


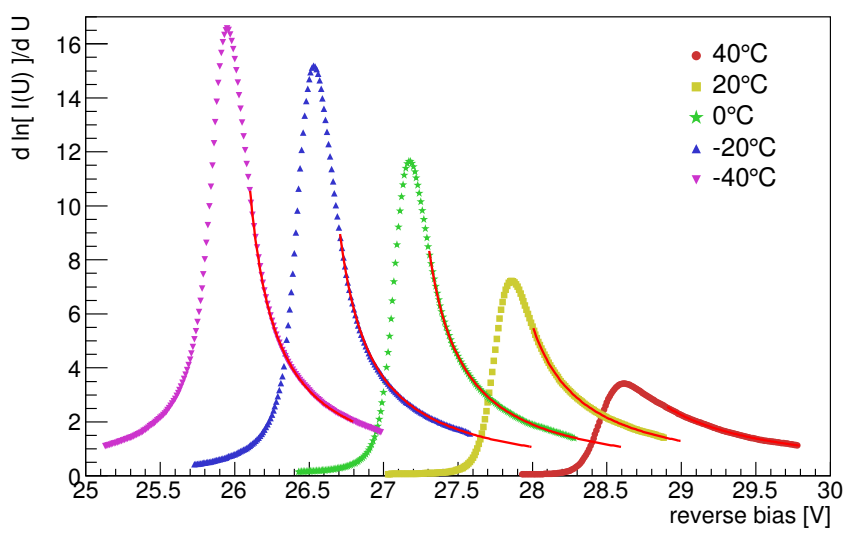

(a) FBK NUV-HD

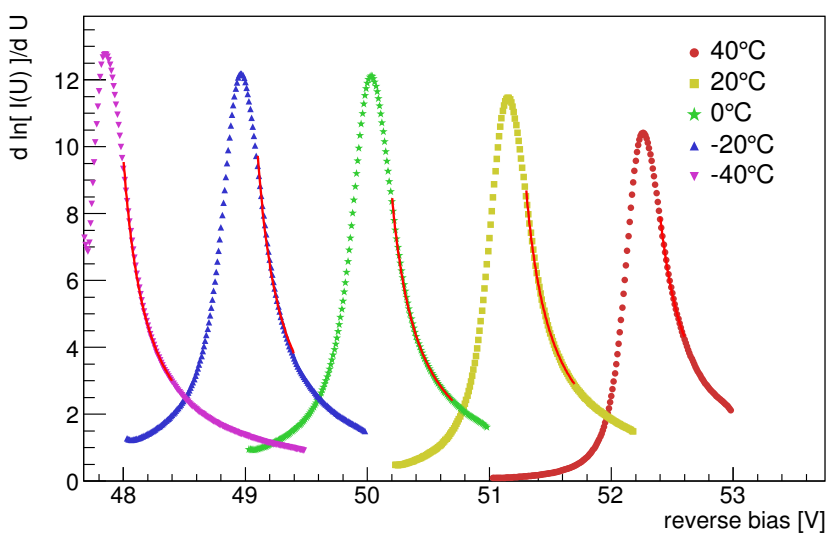

(b) Hamamatsu S13360-3050CS

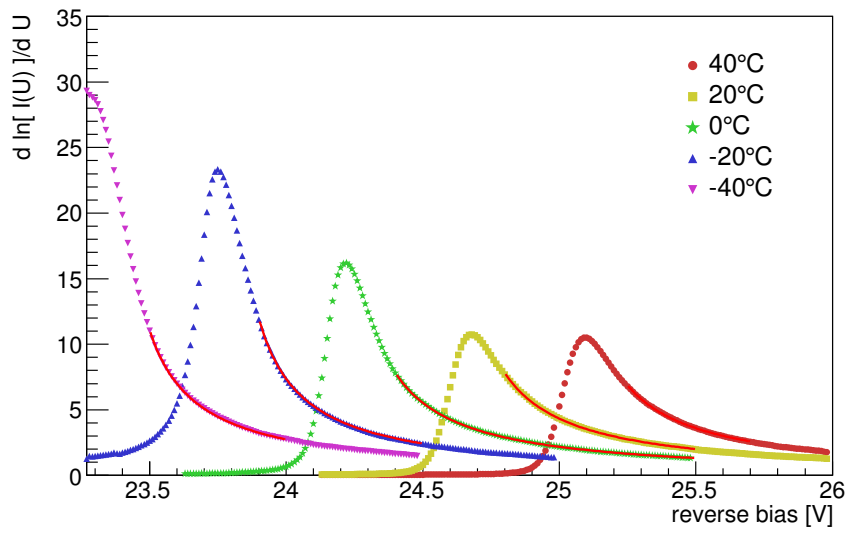

(c) SensL J-series 30035

Figure 16: Derivative of the logarithm of the current around the breakdown voltage. The solid lines are fits to the curves from which the breakdown voltage is determined. tive of the logarithm of the $I V$ curve does not match with the breakdown voltage derived from the gain mea-749 surement. The second reason is that we want to validate 750 the other two methods to derive the breakdown voltage. 751

The model of the simulated $I V$ curve is based on Equa-752 tion 7 extended by the fraction of the dark current, which 753 does not get amplified. The additional term allows one to754

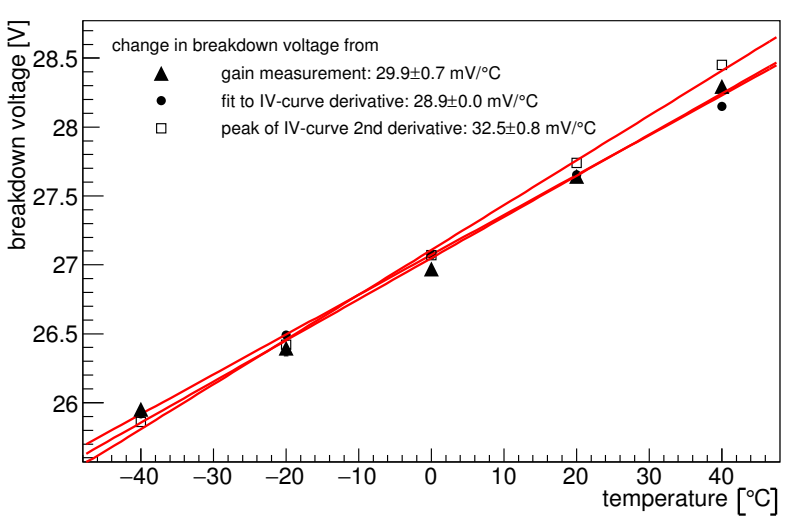

(a) FBK NUV-HD

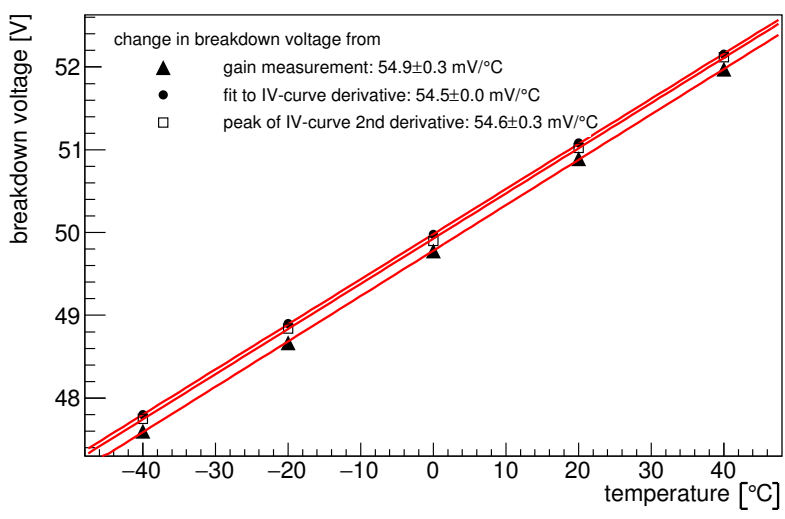

(b) Hamamatsu S13360-3050CS

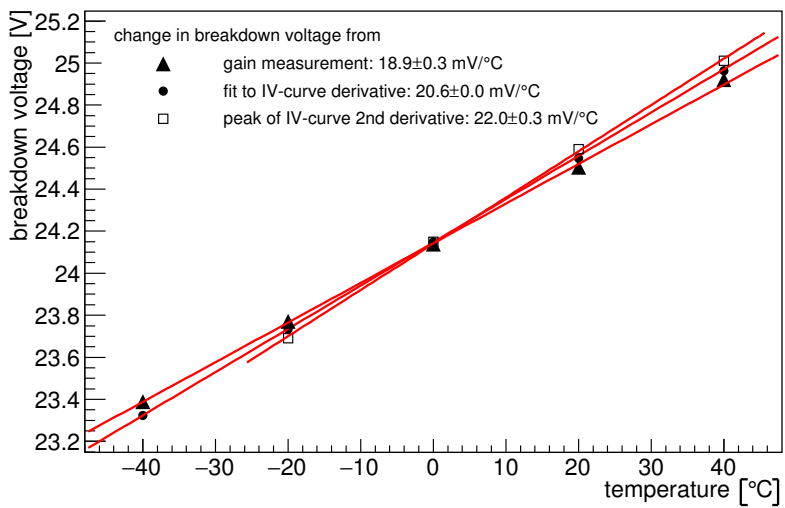

(c) SensL J-series 30035

Figure 17: Breakdown voltage derived from the derivative of the IVcurves (solid dots), the second derivative of the IV-curves (empty squares), and gain measurements (triangles).

simulate the $I V$ curve below the breakdown voltage. As before, contributions from optical crosstalk and afterpulsing have again been neglected. Equation 7 is a model of the absolute current, whereas relevant for the derivation of the breakdown voltage is only the relative change of the current, see Eqn. 10. Therefore, only the relative current 
versus bias curve is simulated:

$$
I_{\mathrm{rel}}\left(U_{\mathrm{rel}}\right)=\frac{I\left(U_{\mathrm{rel}}\right)}{I_{\mathrm{ampl}}}=h+U_{\mathrm{rel}} \cdot G \cdot\left[1-e^{\left(-U_{\mathrm{rel}} / \alpha\right)}\right]
$$

Where the normalization $I_{\text {ampl }}$ is the part of $I_{\mathrm{DC}}+I_{\text {ext }}$ that makes it into the avalanche region and gets amplified. Note that in Eqn. 7 and subsequent equations $I_{\mathrm{DC}}+I_{\text {ext }}$ implicitly denote only the amplified part of the total dark and external generated current. $G$ becomes the product of the cell capacitance and the breakdown voltage and is $6.4 \cdot 10^{6}, 3.5 \cdot 10^{7}$, and $2.5 \cdot 10^{7}$ for the FBK, Hamamatsu, and SensL device, respectively. Note that we restrict ourselves to measurements done at $20^{\circ} \mathrm{C}$. The quantity $h$ is the ratio of the unamplified and amplified part of $I_{\mathrm{DC}}+I_{\text {ext }}$. The value for $h$ is adjusted in the model until the simulated ratio of the currents at $10 \%$ overvoltage and before breakdown matches the data and typically assumes values of 1000 or more.

Cell-to-cell variations of the breakdown voltage are included by simulating 10,000 cells each with a different ${ }^{800}$ breakdown voltage that is randomly picked from a normal ${ }_{81}^{810}$ distribution with a mean of zero and a standard deviation that is a free parameter in the simulation. The simulated $I V$ curve is the sum of the currents of all 10,000 cells.

The last parameter in the simulation is $\alpha$. A small $\alpha$ is expected if the majority of the dark current enters the multiplication region from the front, such as photoelectrons generated by blue photons, and a large $\alpha$ is expected if the ${ }_{816}$ dark current is generated behind the avalanche region, e.g. in the bulk. Increasing $\alpha$ in the model shifts the position of ${ }_{817}$ the maximum of the derivative of the logarithm of the $I V_{818}$ curve towards higher relative overvoltages and can thus be $\mathrm{e}_{819}$ used to tune the simulations to get a match with the data. . 20 A good agreement with measurements is achieved if $\alpha$ is $_{821}$ $0.015,0.05$, and 0.1 for the FBK, Hamamatsu, and SensL $\mathrm{L}_{822}$ devices, respectively. The agreement remains good if $\alpha$ is $_{823}$ varied within the range of values listed for each device $i_{824}$ Table 1.

The width of the peak of the derivative of the logarithm $m_{826}$ of the $I V$ curve is tuned by changing the standard devia- ${ }_{827}$ tion of the cell-to-cell variations of the breakdown voltage. ${ }_{822}$ A value of 0.001 reproduces the FWHM of the measure- ${ }_{829}$ ments of all three SiPMs.

We remark that we did not perform a rigorous tuning ${ }_{831}$ of the model parameters. Therefore, we cannot exclude 832 that a completely different set of model parameters with $_{833}$ different physics implications can equally well reproduce 834 the data. However, we are confident that the model $\operatorname{and}_{835}$ its parameterization is good enough to discuss the validity ${ }_{836}$ of the different methods to extract the breakdown voltage. 837

The simulations confirm that the peak position of the $8 з$ derivative of the logarithm of the $I V$ curve is systemat-839 ically above the breakdown voltage. We also find that 840 fitting the derivative reproduces the true breakdown volt-841 age within $0.1 \%$. The maximum of the second derivatives42 also lies within $0.1 \%$ of the breakdown voltage.

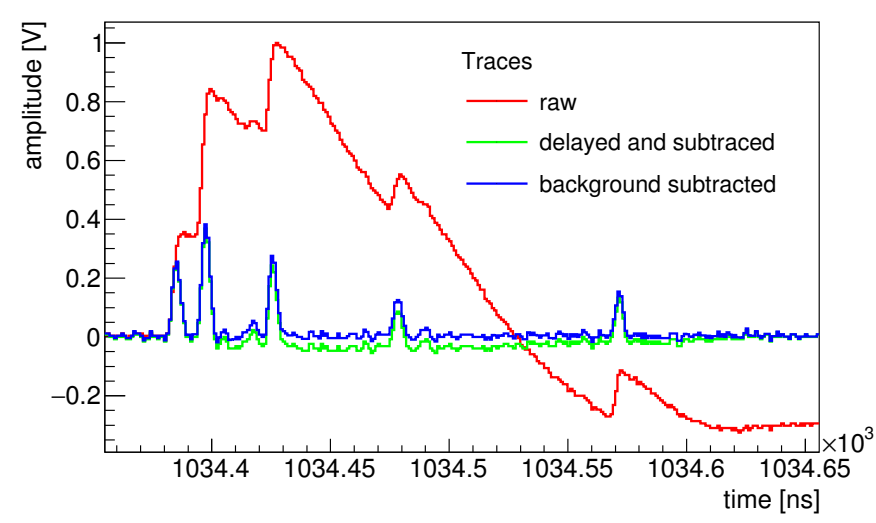

Figure 18: Snapshot of an SiPM trace recorded with $1 \mathrm{GS} / \mathrm{s}$ and 8 bit resolution after amplification (red). The remaining two curves show the trace at two different stages of its processing to reduce the signal widths. See text for details.

Our fitting method and the second-derivative method to extract the breakdown voltage, therefore, seem to be on solid footing. However, we emphasize that the breakdown voltages extracted from the $I V$ curves of the Hamamatsu $\mathrm{SiPM}$ are inconsistent with the ones from the gain vs. bias measurements on the level of $0.4 \%(200 \mathrm{mV})$ for which we do not have an explanation.

\section{Signal trace analysis}

In the remainder of the paper, we discuss the analysis of SiPM signals recorded with the Alazar ATS 9870 digitizer after amplifying the signal with a Mini-Circuits ZFL 500LN+ amplifier and a LeCroy Model 612A amplifier (see Fig. 13). For the absolute calibration of the gain measurement, the SiPM signals were recorded in parallel with a Tektronix TDS 3054C oscilloscope after amplification of the SiPM signals with the Mini-Circuits ZFL $500 \mathrm{LN}+$ preamplifier.

The SiPM signals need to be processed to eliminate the long tails of the individual signals. Fig. 18 shows an example of a recorded SiPM trace before (red) and after (blue) processing. Long tails are a general feature of SiPMs with surface areas larger than $1 \mathrm{~mm}^{2}$ because their terminal capacitance increases with sensor area which, combined with a $50 \mathrm{Ohm}$ input impedance preamplifier, results in long tails. Long tails are also the result of cell recovery times that are less than a few hundred nanoseconds long.

To process the signals, we follow a two-step procedure similar to the approach used in [19]. In the first step, a copy of the original trace is shifted by three nanoseconds and subtracted from the original trace. This step results in a significant shortening of individual SiPM signals down to a full width of about $9 \mathrm{~ns}$. An example of the outcome of this processing step is shown as the green trace in Fig. 18 . A small remaining undershoot is subtracted from the trace by applying a background-subtraction algorithm that is 


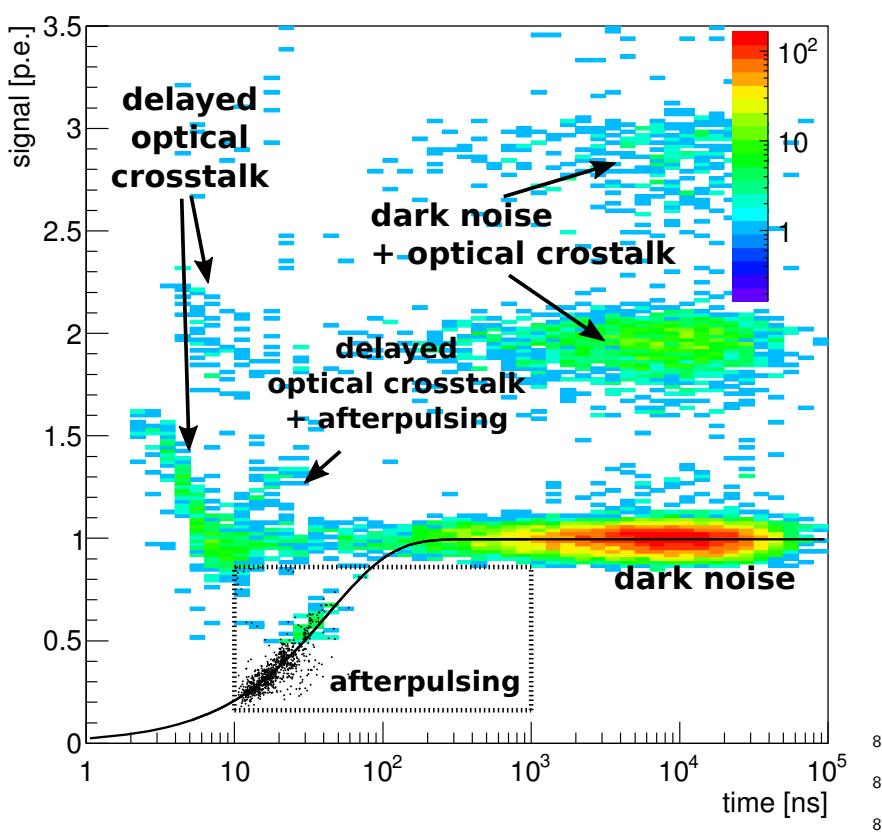

Figure 19: The histogram shows the time difference between two con- ${ }^{876}$ secutive SiPM signals on the $x$-axis and the amplitude of the second ${ }^{87}$ signal on the $y$-axis. Note the logarithmic scale of the $\mathrm{x}$-axis. The $\mathrm{e}^{878}$ colors represent the number of events in each bin on a logarithmic 879 scale. Several populations can be identified and are correspondingly ${ }_{880}$ labeled. implemented in the ROOT analysis framework [20]. The ${ }^{83}$ final result is shown as the blue trace in the figure. $\quad 884$

The general procedure of the signal trace analysis is to $0^{885}$ record randomly triggered $10 \mathrm{~ms}$ long signal traces until ${ }^{886}$ enough statistics are accumulated to reconstruct all pa-887 rameters of interest with high enough precision. The mea-888 surement of the afterpulsing is typically the bottleneck and ${ }^{889}$ defines how many traces need to be recorded. At low tem-890 peratures a dimmed LED is used to increase the $\mathrm{SiPM}^{891}$ signal rate and thus speed up the afterpulsing measure-892 ment. Measurements of the dark rate are made with the LED turned off.

After a trace is processed, all SiPM signals with an amplitude of at least 0.5 photoelectrons (p.e.) are identified, ${ }_{895}{ }_{994}$ erated when one cell of an SiPM breaks down. The amplitudes and times of the identified signals are then used ${ }_{898}^{897}$ to extract the SiPM parameters (similar to how it is described in [19]).

An illustrative example of the type of information that ${ }_{900}^{900}$ can be extracted from the amplitudes and times is given ${ }_{902}$ in Fig. 19. It is a two-dimensional histogram that has the ${ }_{903}$ time difference between two consecutive signals on the $\mathrm{x}^{-903}$ axis and the amplitude of the second signal in units of p.e. on the y-axis. The color gives the number of events per bin. In this figure only signal pairs have been selected in which ${ }_{907}$ the first signal has an amplitude of one photoelectron.

A number of different populations can easily be identified. The biggest population is made up by signals in i.e. signals with at least half the amplitude that is gen- ${ }_{896}$

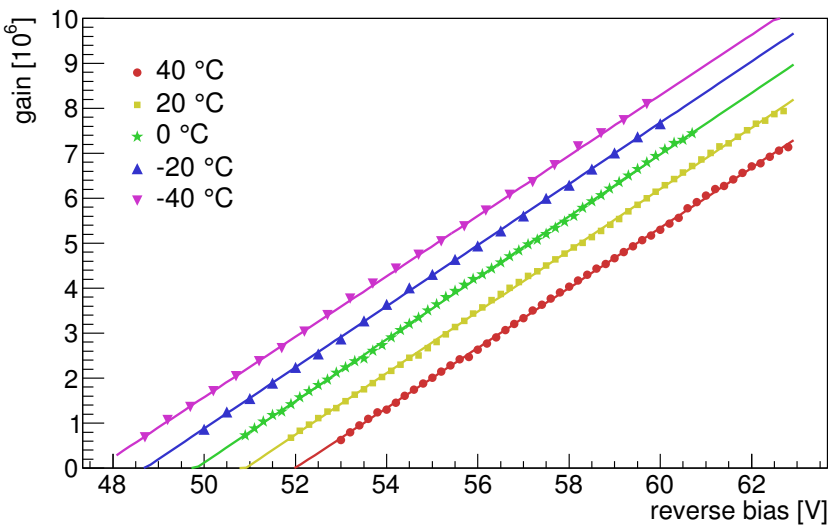

Figure 20: Gain vs. bias for the Hamamatsu SiPM for five different temperatures.

which only one cell of the SiPM fires. That population peaks at a time difference of $\approx 10 \mu \mathrm{s}$, which is the expected average time difference between two uncorrelated dark count signals, i.e. the inverse of the dark count rate for that specific device and temperature. The bands above that population are from signals where one cell fires due to an uncorrelated dark count, and one or two additional cells fire in coincidence due to direct optical crosstalk.

To the left of the main blob is a smaller population that is due to delayed optical crosstalk signals. The amplitudes of the delayed optical-crosstalk signals to the very left depend on the time when the signal appears because there is significant overlap with the preceding signal, and the signal-extraction algorithm is not able to properly handle the overlap. ${ }^{4}$

Also visible are afterpulsing events that generate a second signal from the same cell before it is fully recharged. The solid black line shows a fit to the afterpulsing events in the dashed box and is used to measure the recovery time of one cell.

\subsection{Gain, Cell Capacitance and Breakdown Voltage}

The first information extracted from the signal amplitudes is the signal charge in units of electrons, which is commonly referred to as the gain of an SiPM. The amplitudes of signals between 0.5 and 1.5 p.e. are averaged and then converted into signal charge. For this conversion, a separate calibration of the entire signal chain was performed for each SiPM.

In the first step of the calibration, the average single p.e. amplitude was read off a Tektronix TDS 3054C oscilloscope at a temperature of $-20^{\circ} \mathrm{C}$ and at two different bias voltages after amplification of the raw signals with a Mini-Circuits ZFL-500LN+. The uncertainty in reading the amplitude off the oscilloscope is $0.2 \%$ and dominates the uncertainty of the absolute gain and breakdown

\footnotetext{
${ }^{4}$ The width of one signal is $9 \mathrm{~ns}$ after a trace is processed.
} 
voltage measurement. In the second step, the signal amplitudes are divided by the gain of the amplifier $(30 \mathrm{~dB})$. In the third step, the calibrated amplitudes are multiplied with the integral of the normalized raw signal shape, ${ }^{5}$ thus obtaining two absolute gain measurements. These two absolute gain measurements and the average single-cell amplitudes that were extracted from the processed traces at the same bias and temperature are then used to define a linear transformation from processed signal amplitude to absolute charge.

An example of a calibrated gain measurement is shown in Fig. 20. The solid lines are linear fits to the data. A closer inspection of the data points reveals small residuals with respect to the fits, which can be attributed to nonlinearities in the front-end amplifier of the digitizer.

The linear dependence of the gain on bias can be explained in the small-signal model of SiPMs where the cell of an SiPM is represented by a capacitance $C_{\text {cell }}$ that is discharged to the breakdown voltage in a breakdown. The total charge $G$ of the signal is then

$$
G=C_{\text {cell }} \cdot\left(U-U_{\mathrm{BD}}\right) .
$$

If $G$ is given in units of electrons, it is usually referred to as the gain of the device, which is the definition of $G$ we adopt in this paper.

Based on Equation 12 the breakdown voltage can be measured from the gain vs. bias curve as the voltage where the gain is zero. The determined breakdown voltage is shown in Fig. 17 together with those extracted from the $I V$-curves.

The cell capacitance $C_{\text {cell }}$ is given by the slope of the gain vs. bias measurement and is shown in Fig. 21. For the Hamamatsu and the FBK SiPM the cell capacitance remains constant, whereas a $5 \%$ change is seen in the SensL SiPM between $-40^{\circ} \mathrm{C}$ and $40^{\circ} \mathrm{C}$. The gain vs. bias curves are well described by linear functions, and aside from the residuals that can be attributed to the digitizer, no further deviation from linearity is observed that would point to a dependence of the cell capacitance on bias for any of the tested devices.

\subsection{Dark count rates}

The dark count rates are measured by counting all signals with an amplitude larger than 0.5 p.e. and dividing that number by the total duration of all analyzed traces. Included in this measurement are, therefore, thermal generated dark counts as well as delayed optical crosstalk and afterpulsing. However, the latter two contribute only mi-959 nor to the total dark count rate as they are less than $2 \%$ at ${ }_{960}$ $90 \%$ breakdown probability. Two pulses have to be at least $\approx 3$ ns apart in order to be identified as separate signals. ${ }^{961}$

Fig. 22 shows the dark count rates per one square millimeter sensor area for all temperatures and for all three ${ }^{962}$

5 The signal shape was normalized to a peak amplitude of one. 965

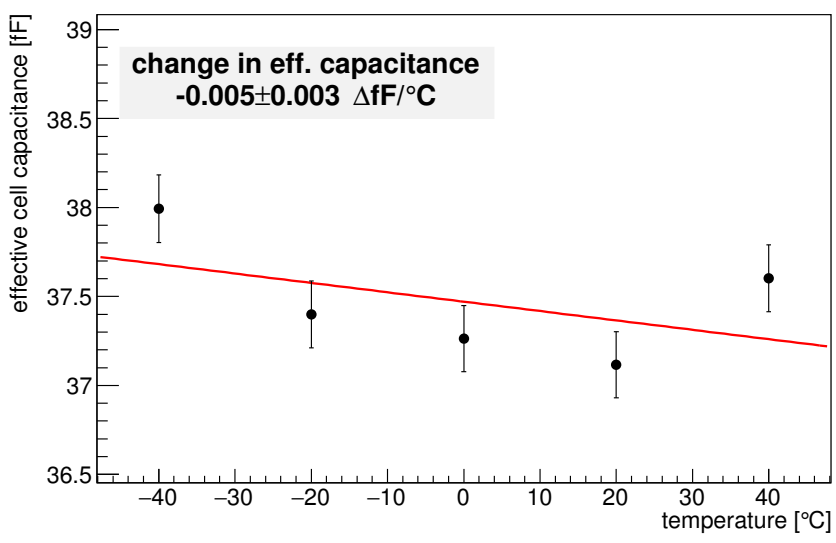

(a) FBK NUV-HD

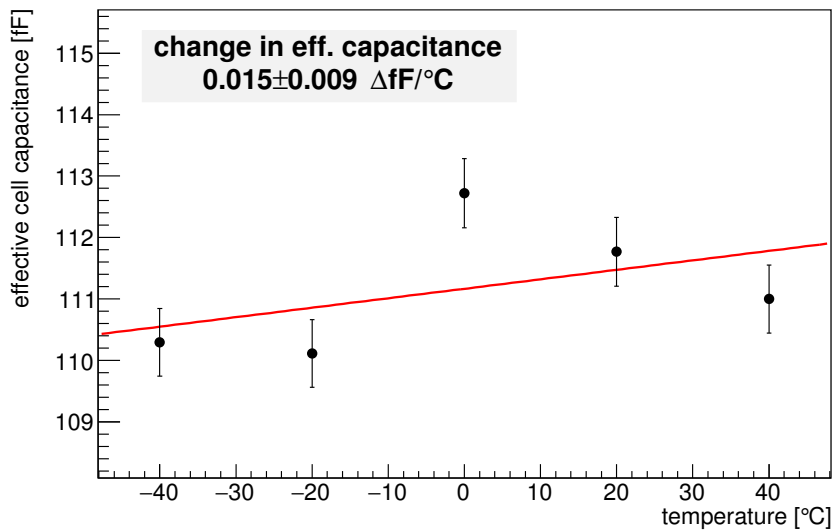

(b) Hamamatsu S13360-3050CS

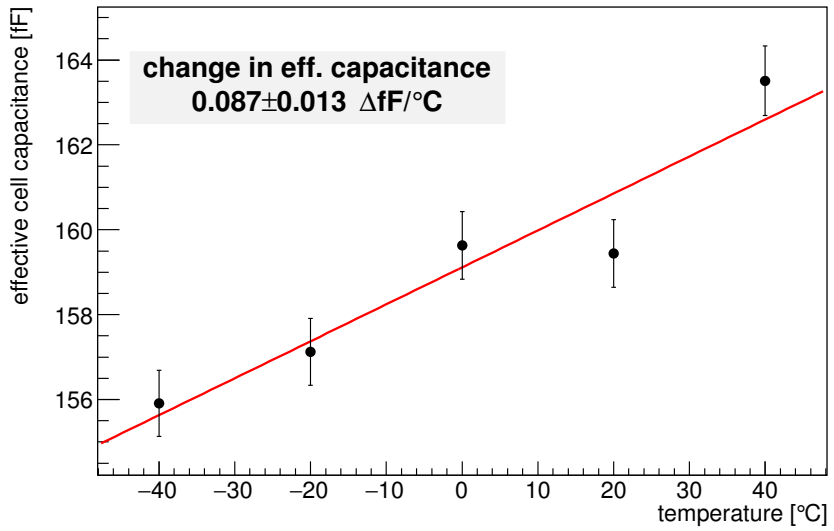

(c) SensL J-series 30035

Figure 21: Cell capacitance.

devices. The solid lines are fits to the data with the function

$$
D C\left(U_{\mathrm{rel}}\right)=e^{a+b \cdot U_{\mathrm{rel}}} \cdot\left[1-e^{\left(-U_{\mathrm{rel}} / \alpha\right)}\right],
$$

where the last term is the breakdown probability and is only used in the fit of the dark rate measurement of the Hamamatsu SiPM. For the SensL and FBK SiPMs the dark-rate measurements start at an overvoltage where the 


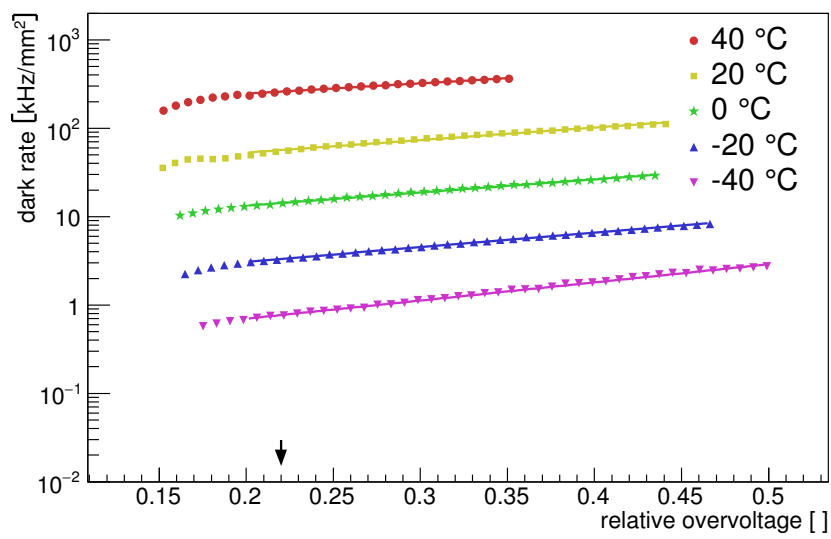

(a) FBK NUV-HD

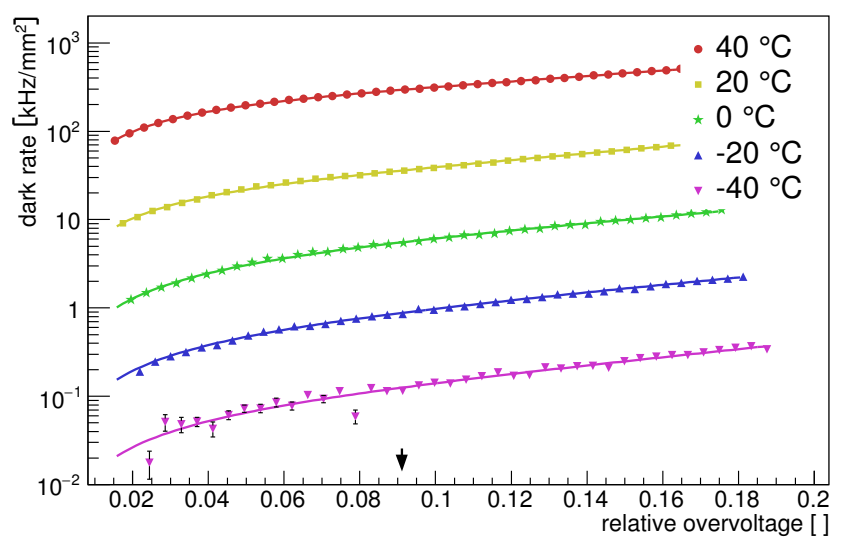

Table 2: Best Fit Values Obtained From the Fit of the Dark Rate Measurements Shown in Fig. 22.

\begin{tabular}{c|c|c|c|c} 
Dev. & Temp. & $a$ & $b$ & $\alpha\left[10^{-2}\right]$ \\
\hline \hline FBK & $-40^{\circ} \mathrm{C}$ & $-1.30 \pm 0.01$ & $4.72 \pm 0.02$ & \\
& $-20^{\circ} \mathrm{C}$ & $0.368 \pm 0.003$ & $3.79 \pm 0.01$ & \\
& $0^{\circ} \mathrm{C}$ & $1.91 \pm 0.01$ & $3.41 \pm 0.01$ & \\
& $20^{\circ} \mathrm{C}$ & $3.31 \pm 0.01$ & $3.26 \pm 0.01$ & \\
& $40^{\circ} \mathrm{C}$ & $4.97 \pm 0.01$ & $2.68 \pm 0.01$ & \\
\hline Ham. & $-40^{\circ} \mathrm{C}$ & $-2.9 \pm 0.1$ & $10.1 \pm 0.7$ & $4 \pm 1$ \\
& $-20^{\circ} \mathrm{C}$ & $-0.84 \pm 0.03$ & $9.2 \pm 0.2$ & $4.3 \pm 0.2$ \\
& $0^{\circ} \mathrm{C}$ & $1.11 \pm 0.02$ & $8.2 \pm 0.1$ & $4.5 \pm 0.1$ \\
& $20^{\circ} \mathrm{C}$ & $2.86 \pm 0.01$ & $8.43 \pm 0.06$ & $2.8 \pm 0.1$ \\
& $40^{\circ} \mathrm{C}$ & $5.100 \pm 0.003$ & $6.83 \pm 0.02$ & $2.7 \pm 0.1$ \\
\hline SensL & $-40^{\circ} \mathrm{C}$ & $-2.56 \pm 0.01$ & $9.22 \pm 0.04$ & \\
& $-20^{\circ} \mathrm{C}$ & $-0.86 \pm 0.01$ & $9.19 \pm 0.03$ & \\
& $0^{\circ} \mathrm{C}$ & $0.92 \pm 0.01$ & $8.65 \pm 0.02$ & \\
& $20^{\circ} \mathrm{C}$ & $2.662 \pm 0.001$ & $7.92 \pm 0.01$ & \\
& $40^{\circ} \mathrm{C}$ & $5.055 \pm 0.001$ & $6.71 \pm 0.01$ & \\
\hline
\end{tabular}

(b) Hamamatsu S13360-3050CS

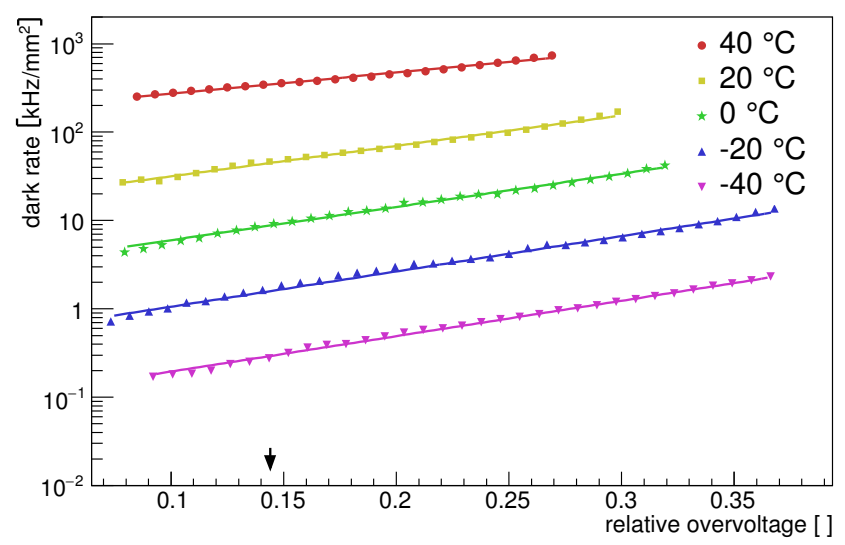

(c) SensL J-series 30035

Figure 22: Dark count rates. The arrow marks the nominal operating bias of each device.

breakdown probability is already close to $90 \%$ (check the996 position of the arrow). The turnover in the data for the997 FBK device occurs because the small cell capacitance re-998 sults in signals too small to be reliably detected with ourg9s signal chain at low overvoltages. The results of the fits are000 shown in Table 2 . The $\alpha$ values extracted for the Hamał001 matsu SiPM are consistent with the $\alpha$ extracted from theoo PDE measurements (s. Table 1) for short photon wave 4003 lengths, which indicates that the majority of the dark noise enters the avalanche region from the surface of the device.

The rates in Fig. 22 are shown versus relative overvoltage. For a fixed relative overvoltage, any change in the dark rate with temperature can be attributed to changes in the thermal generation of charge carriers. Fig. 23 shows how the dark count rate changes with temperature for a fixed overvoltage relative to the dark count rate at $40^{\circ} \mathrm{C}$ and averaged over the operating voltage range at $40^{\circ} \mathrm{C}$. The relative change in dark count rate with temperature for all three devices is well described by $e^{a+b \cdot T}$. The change in temperature needed to change the dark count rate by a factor of two is stated in the inserts in the figure.

\subsection{Optical crosstalk}

Optical crosstalk (OC) is the correlated firing of cells due to photons emitted in the breakdown of one cell. Any of these photons can initiate the breakdown of a neighboring cell. Two types of optical crosstalk can be distinguished. Direct OC is due to crosstalk photons that get absorbed in the active volume of a neighboring cell and cause the breakdown of that additional cell, which is quasi-simultaneous to the first one. Delayed OC is due to crosstalk photons that convert in the non-depleted bulk. In this case the generated charge carrier has to first diffuse into the active volume of the cell $[19,21,22]$. The diffusion process introduces a measurable time delay between the breakdown of the first cell and the breakdown of the second cell.

Measurements of the direct $\mathrm{OC}$ are presented in this section and the delayed OC measurements are discussed 


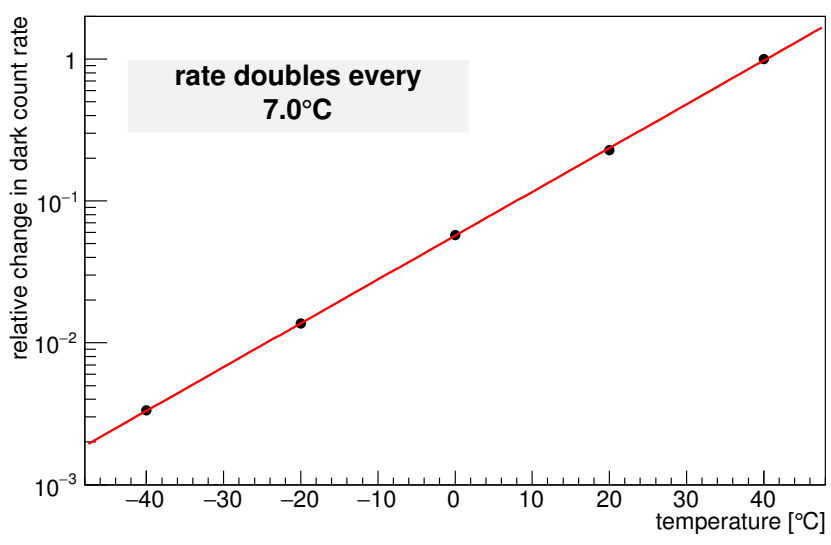

(a) FBK NUV-HD

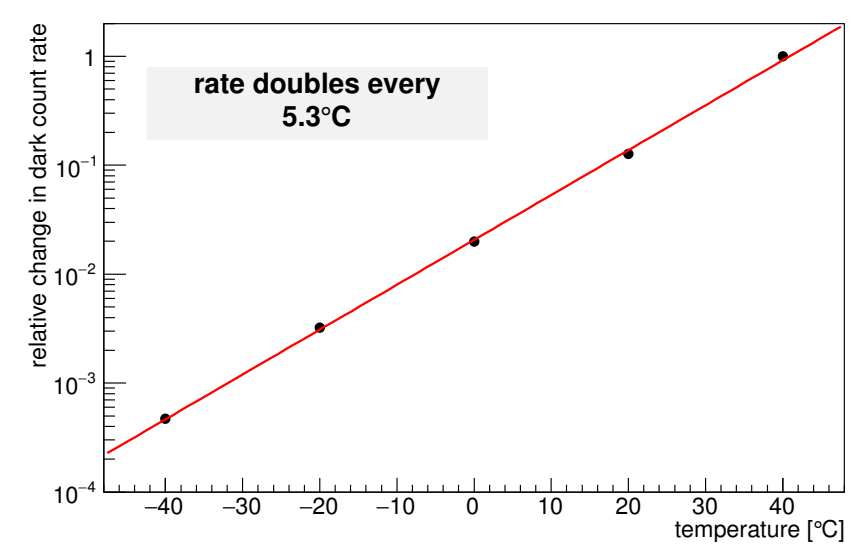

(b) Hamamatsu S13360-3050CS

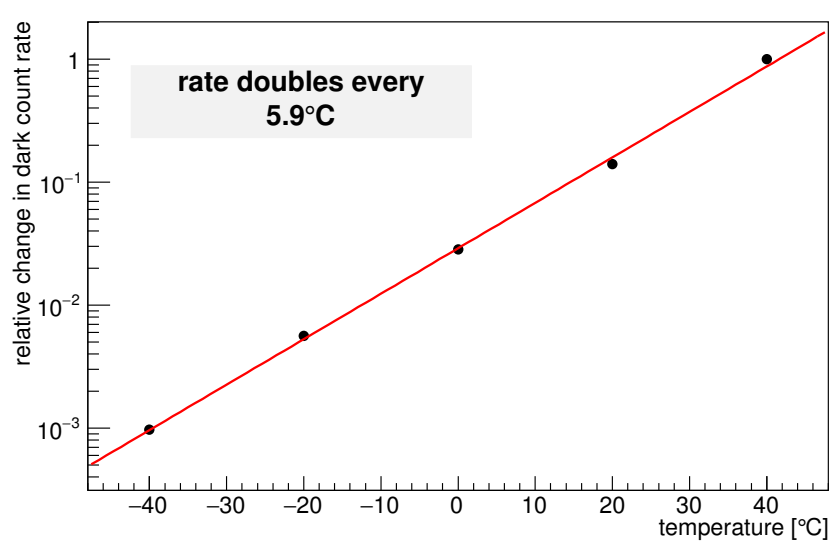

(c) SensL J-series 30035

Figure 23: Relative change in dark count rates.

together with afterpulsing measurements in the next $\sec _{\overline{1033}}^{1032}$ tion.

Direct OC is extracted from the pulse height distribu+035 tion of the SiPM signals. Fig. 24 shows an example of such a distribution where events can be clearly identified 037 that are due to 1,2 , or 3 cells firing simultaneously. The small peak on the left is due to afterpulses, which are the same events that are also marked as afterpulses in Fig. $199_{1040}$

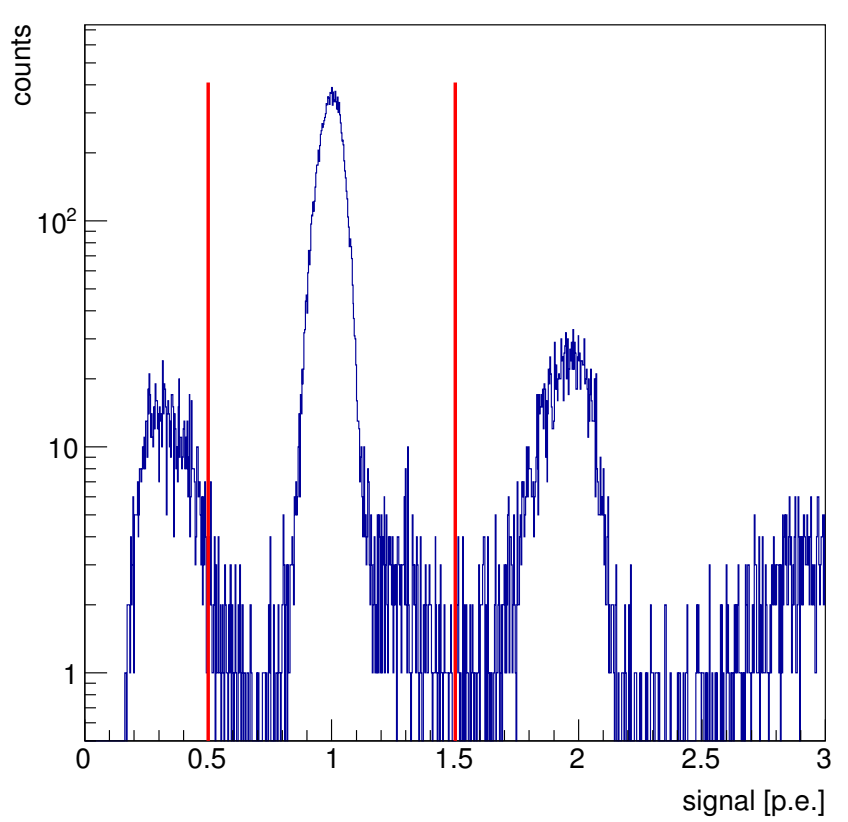

Figure 24: Example of a pulse height distribution of signals from the SensL device. The vertical line at 1.5 p.e. marks the boundary between signals in which only one cell fired (left) and more than one (right). The small peak at the left is due to afterpulsing events that can also be identified in Fig. 19. Only signals with an amplitude of at least 0.5 p.e. are used in the optical crosstalk analysis. 


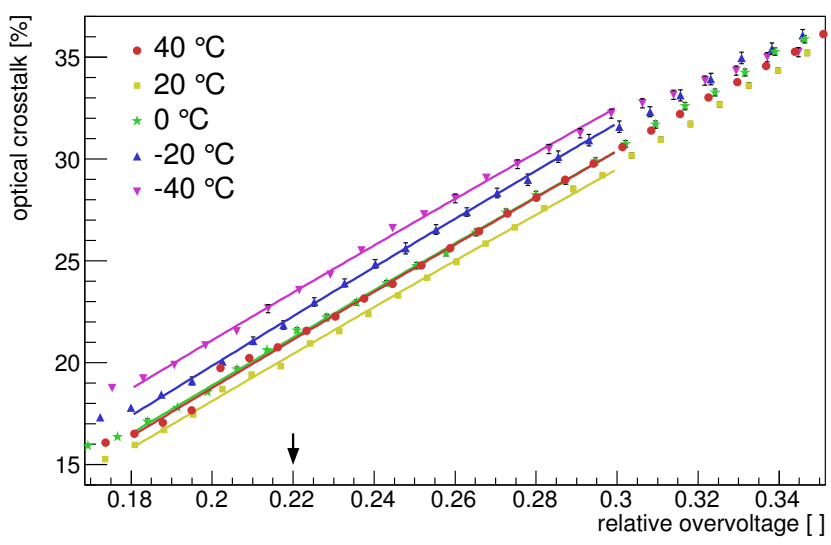

(a) FBK NUV-HD

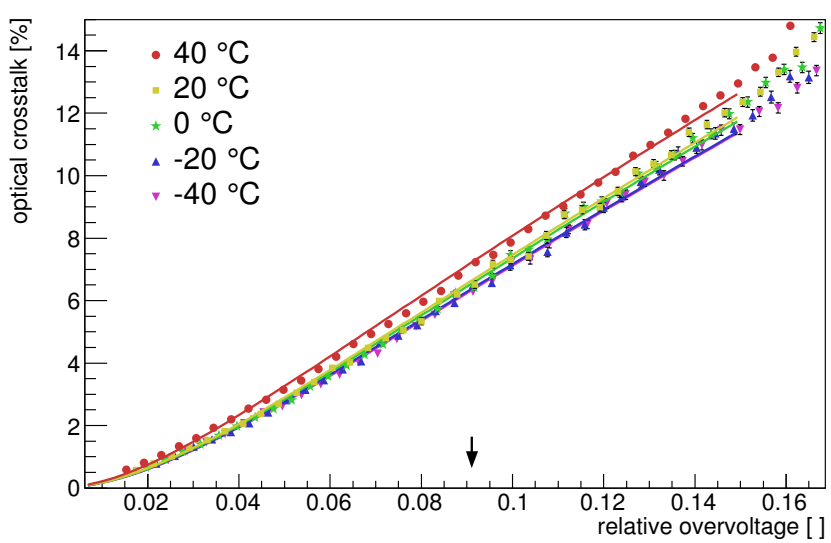

(b) Hamamatsu S13360-3050CS

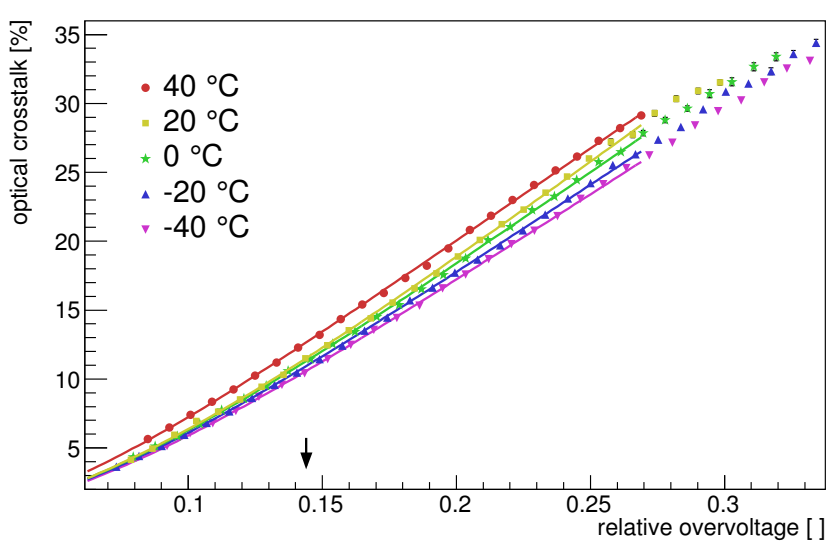

(c) SensL J-series 30035
Table 3: Best Fit Values Obtained From Fitting the Direct Optical Crosstalk Measurements Shown in Fig. 25. The Last Column Shows the Probability That a Photon Emitted in a Breakdown Results in a Breakdown of a Neighboring Cell.

\begin{tabular}{c|c|c|c} 
Device & Temp. & $\alpha$ & OC efficiency $\gamma$ \\
\hline FBK & $-40^{\circ} \mathrm{C}$ & $0.059 \pm 0.002$ & $0.590 \pm 0.002$ \\
& $-20^{\circ} \mathrm{C}$ & $0.082 \pm 0.004$ & $0.584 \pm 0.005$ \\
& $0^{\circ} \mathrm{C}$ & $0.085 \pm 0.002$ & $0.551 \pm 0.003$ \\
& $20^{\circ} \mathrm{C}$ & $0.092 \pm 0.001$ & $0.531 \pm 0.002$ \\
& $40^{\circ} \mathrm{C}$ & $0.089 \pm 0.001$ & $0.528 \pm 0.001$ \\
\hline Hamamatsu & $-40^{\circ} \mathrm{C}$ & $0.040 \pm 0.001$ & $0.079 \pm 0.001$ \\
& $-20^{\circ} \mathrm{C}$ & $0.040 \pm 0.001$ & $0.076 \pm 0.001$ \\
& $0^{\circ} \mathrm{C}$ & $0.041 \pm 0.001$ & $0.076 \pm 0.001$ \\
& $20^{\circ} \mathrm{C}$ & $0.039 \pm 0.001$ & $0.076 \pm 0.001$ \\
& $40^{\circ} \mathrm{C}$ & $0.034 \pm 0.001$ & $0.078 \pm 0.001$ \\
\hline SensL & $-40^{\circ} \mathrm{C}$ & $0.161 \pm 0.001$ & $0.129 \pm 0.002$ \\
& $-20^{\circ} \mathrm{C}$ & $0.160 \pm 0.001$ & $0.127 \pm 0.001$ \\
& $0^{\circ} \mathrm{C}$ & $0.162 \pm 0.001$ & $0.130 \pm 0.002$ \\
& $20^{\circ} \mathrm{C}$ & $0.168 \pm 0.001$ & $0.137 \pm 0.002$ \\
& $40^{\circ} \mathrm{C}$ & $0.154 \pm 0.001$ & $0.105 \pm 0.001$ \\
\hline
\end{tabular}

is given by the breakdown probability $1-\exp \left(-U_{\text {rel }} / \alpha\right)$. Combining all factors, the $\mathrm{OC}$ as a function of relative overvoltage becomes

$$
O C\left(U_{\mathrm{rel}}\right)=f \cdot C_{\mathrm{eff}} \cdot U_{\mathrm{rel}} \cdot U_{\mathrm{BD}} \cdot \gamma \cdot\left[1-e^{\left(-U_{\mathrm{rel}} / \alpha\right)}\right]
$$

Figure 25: Direct optical crosstalk. The arrow marks the nominal operating bias of each device. stood in the following way. The number of photons emit1069 ted in the breakdown of one cell is $f \cdot C_{\text {eff }} \cdot \Delta U$, where $f$ is ${ }^{070}$ about $3 \cdot 10^{-5}$ photons per electron in the avalanche $[21]_{071}$ and $C_{\text {eff }} \cdot \Delta U$ is the gain in units of electrons. Each emittedo72 photon has a probability $\gamma$ to absorb in the active volume 073 of a neighboring cell and generate a charge carrier. The 074 likelihood of that charge carrier to initiate a breakdown 075
The overvoltage dependence of the OC can be under ${ }^{1068}$ cillition

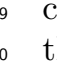
systevice are likely affected by the above mentioned the peaks in the pulse height distriution and thus should be interpreted with caution. 


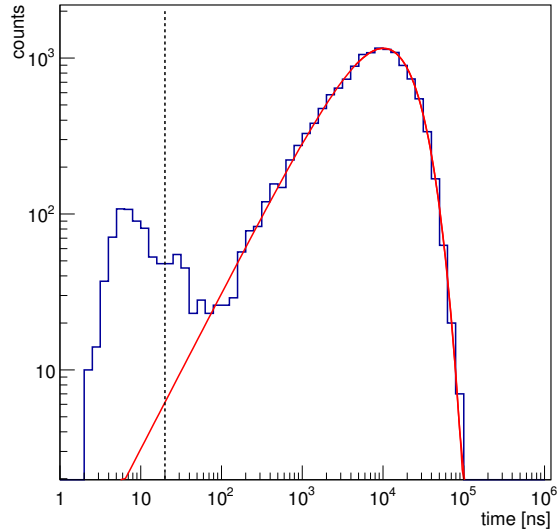

(a) Only next pulse

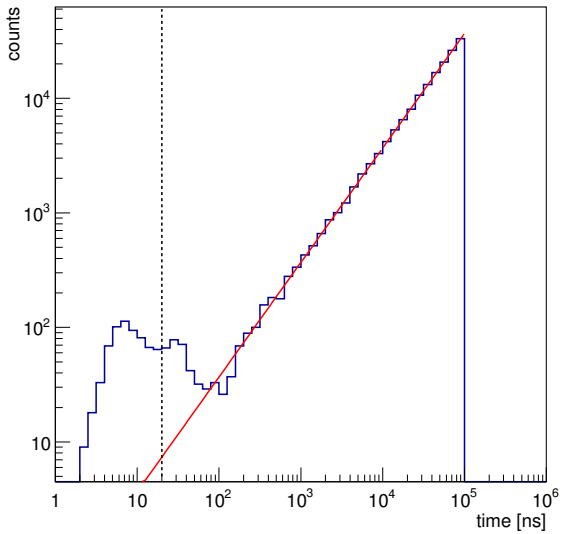

(b) All following pulses

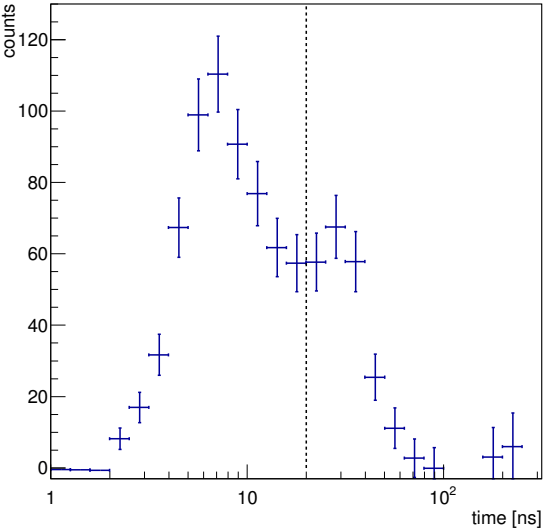

(c) Residuals

Figure 26: Example of distributions of time differences between two pulses from the SensL device. See text for details.

\subsection{Afterpulsing and Delayed Optical Crosstalk}

1114

Afterpulsing and delayed OC both produce signals that ${ }^{115}$ are correlated in time with respect to a previous $\mathrm{SiPM}^{116}$ signal. Both effects are quantified by selecting SiPM sig-117 nals with amplitudes between 0.5 and 1.5 p.e. and record ${ }^{1118}$ ing the time to the next signal. Fig. 26a shows an exam-1119 ple of the distribution of the time differences. The main ${ }^{120}$ peak is due to uncorrelated, Poisson-distributed dark-noise $\mathrm{e}^{121}$ counts. The position of the peak is at the average time ${ }^{122}$ difference between two dark counts, which is equal to the ${ }^{123}$ inverse of the dark-count rate. We note that the binning.124 of the histograms is logarithmic, and as a result of the $\operatorname{bin}^{1225}$ ning, the Poisson distribution takes the form $a \cdot t \cdot \exp (-t / \tau)^{126}$ instead of a pure exponential function. The main peak $\mathrm{i}^{127}$ well fit with a Poisson distribution, and the residuals due ${ }^{128}$ to delayed optical crosstalk and afterpulses at small time ${ }^{129}$ differences are clearly visible.

For the extraction of the delayed $\mathrm{OC}$ and afterpulsing ${ }^{131}$ probabilities, however, we histogram not only the time dif ${ }^{1132}$ ference between the first and the next pulse, but all fol ${ }^{1133}$ lowing pulses up to a time difference of $100 \mu \mathrm{s}$. In this way134 we eliminate the need to consider cases in which an after ${ }^{1135}$ pulse or delayed OC signal is missed because of an earlierriso dark count. Fig. 26b shows the corresponding pulse height137 distribution. The Poisson-distributed dark counts followr138 a line through the origin now. The fit of the distribution ${ }^{139}$ with a line was performed between $10 \mu \mathrm{s}$ and $100 \mu \mathrm{s}$. The140 figure to the right shows the residuals between the data141 and the fit, which are due to delayed OC and afterpulses 1142

The residuals consist of two components. The left $\operatorname{com}^{1143}$ ponent is due to delayed $\mathrm{OC}$, and the right is due to af $\mathrm{f}_{1144}$ terpulses. The two components are better visible in the145 amplitude vs. time distribution shown in Fig. 19. Delayed ${ }^{146}$ OC produces signals with amplitudes of 1 p.e. or larger ${ }_{1147}$ whereas afterpulses have amplitudes between 0 and $1 . \quad{ }_{1148}$

For the measurement of the afterpulsing probability, we $e_{19}$ select all the events in the residual distribution that are to150 the right-hand side of the time delay when the amplitude 151 of afterpulses reaches 0.5 p.e. Residuals with shorter time delays are assumed to be due to delayed OC. The vertical lines in Fig. 26 give an example of where the boundary between the two components is placed for the SensL SiPM. The dividing time delay is $50 \mathrm{~ns}$ for the FBK, $17 \mathrm{~ns}$ for the Hamamatsu, and $20 \mathrm{~ns}$ for the SensL device.

The method is robust but does not provide a clean separation between the two components. A more rigorous approach would also include the amplitude information, which allows a clear separation between the two components (see Fig. 19). Such an approach would also allow extracting the trapping times of the afterpulses. We did not implement such an analysis because our method to extract the amplitudes and times becomes increasingly inefficient if two pulses are separated by less than $10 \mathrm{~ns}$. This inefficiency introduces a considerable systematic effect and results in an underestimation of the delayed optical crosstalk, which dominates the uncertainty in any of our measurements.

Figures 27 and 28 show the delayed OC and afterpulsing probabilities, respectively. At their respective operating voltages all devices have a probability for delayed OC of about $2 \%$. The afterpulsing probability is less than $2 \%$ for the Hamamatsu SiPM and less than $1 \%$ for the FBK and SensL SiPM. Again we note that the delayed OC has to be understood as a lower limit due to the inefficiencies of extracting pulses with time differences that are less than $10 \mathrm{~ns}$. The afterpulsing probabilities on the other hand are likely overestimated by about $20 \%$ because of the hard cut that is applied in the residuals to divide the two components. The best separation between the two components is achieved in the measurement of the Hamamatsu device and is thus the least affected by an overspill of OC events.

From the point of view of judging the performance of the three SiPMs in an application, the afterpulsing and delayed OC probabilities at the operating voltages are sufficiently low that it is in fact not necessary to perform a more detailed analysis of, for example, the afterpulsing 


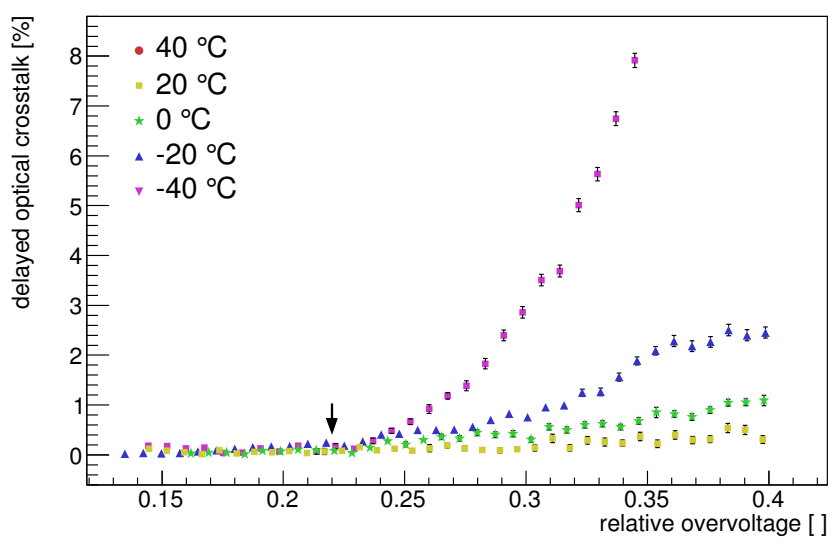

(a) FBK NUV-HD

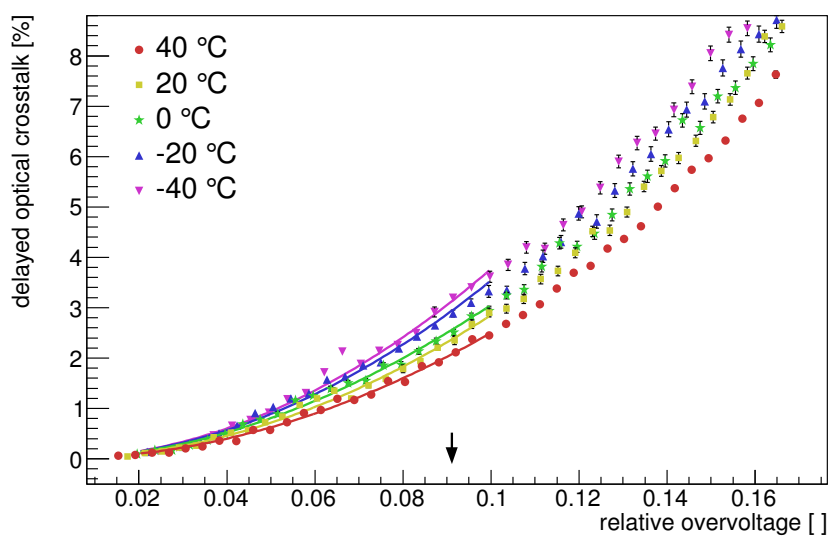

(b) Hamamatsu S13360-3050CS

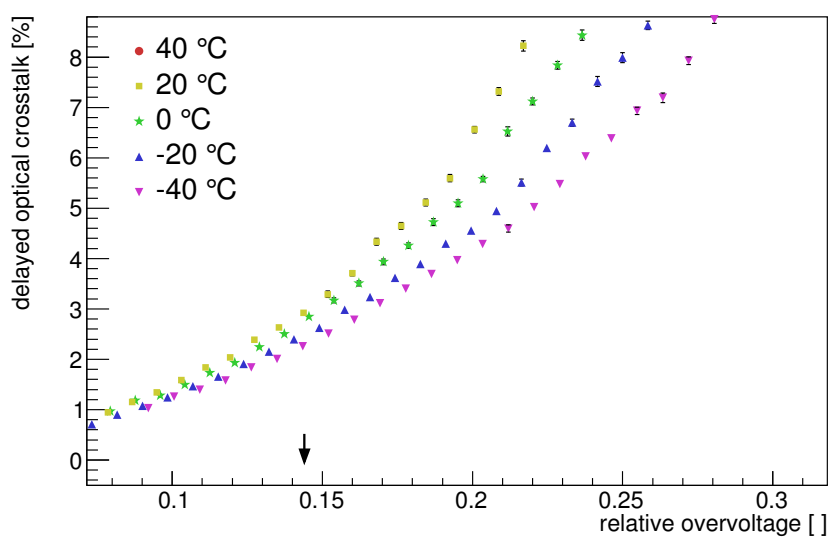

(c) SensL J-series 30035

Figure 27: Delayed optical crosstalk. The arrow marks the nominal operating bias of each device. trapping time constants.

The overvoltage dependence of the delayed OC can be $\mathrm{e}^{161}$ expected to be described in the same way as the direct $\mathrm{OC}_{1162}$ i.e., with Equation 14. Fits to the Hamamatsu data are shown in the Figure 27. However, due to the inefficiency163 in our pulse-extraction algorithm, we could not extract 164 meaningful parameters from the fit, which is also reflected ${ }_{165}$ by a poor probability of the fit.

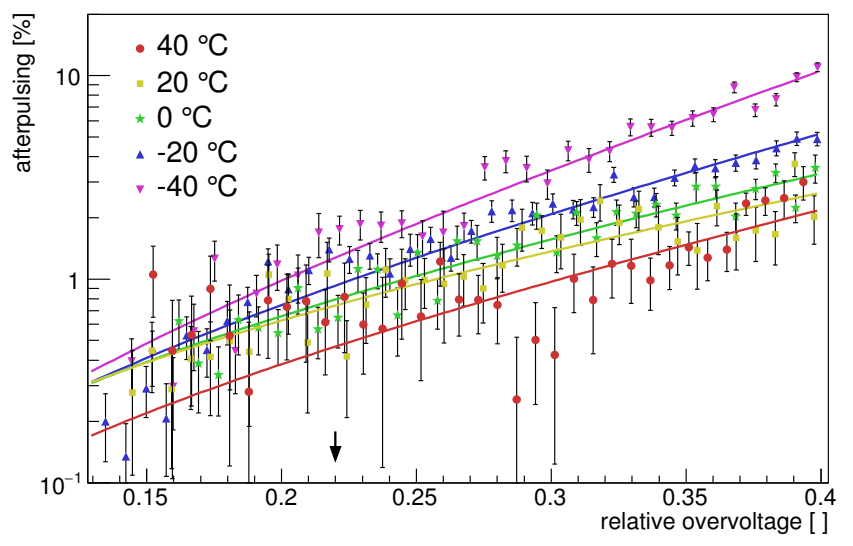

(a) FBK NUV-HD

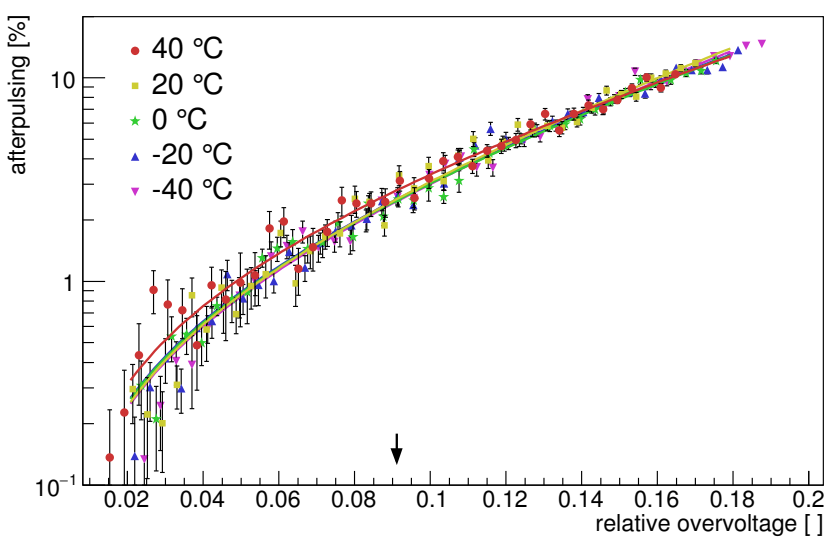

(b) Hamamatsu S13360-3050CS

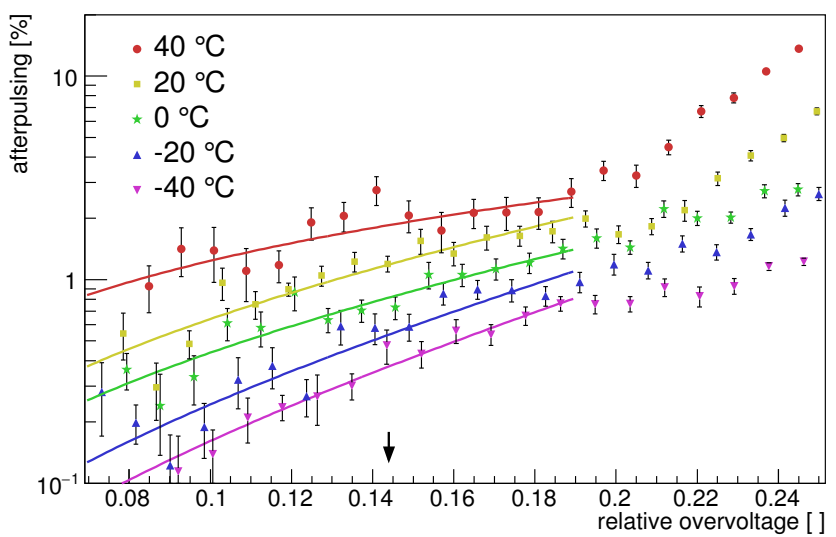

(c) SensL J-series 30035

Figure 28: Afterpulsing. The arrow marks the nominal operating bias of each device.

The afterpulsing vs. overvoltage data are fit with the function

$$
A P\left(U_{\mathrm{rel}}\right)=A \cdot e^{\left(U_{\mathrm{rel}} / \delta\right)} \cdot\left[1-e^{\left(-U_{\mathrm{rel}} / \alpha\right)}\right]
$$

where $A$ is a normalization, and the second term describes the bias dependence of the afterpulsing probability. The last term has to be understood as an effective breakdown probability because it averages over all possi- 
ble times when afterpulses can happen during the recovery of a cell. Because individual trapping times are exponentially distributed, the majority of the trapped charges are released shortly after the breakdown of a cell has stopped. This means that the breakdown probability is small at the time when most afterpulse are released and $\alpha$, therefore, expected to be large.

The afterpulsing as function of bias does not show a dependence on temperature for the Hamamatsu SiPM. We note that trapping time constants decrease exponentially with increasing temperature. It is thus expected that afterpulsing decreases with increasing temperature because more trapped carriers are released before the cell recovers to a meaningful breakdown probability. The expected temperature behavior is observed in the FBK device but not in the SensL device. We cannot rule out that the observed behaviour is due to a contamination of afterpulses with delayed optical crosstalk events.

For the FBK, Hamamatsu, and SensL SiPMs, the fit values averaged over all temperatures for $\alpha$ are 80, 80, and 100, respectively. For $\delta$ they are 0.2, 0.09, and 0.15, respectively. The uncertainties are fairly large and hide any temperature dependencies.

\subsection{Cell Recovery Times}

The last quantity measured is the cell recovery time. Cell recovery times can be measured by flashing an SiPM with two fast consecutive pulses and recording how the second SiPM signal amplitude changes as a function of the time difference between the two pulses. The recovery time can also be measured by analyzing the amplitude vs. time characteristics of afterpulses, which is expected to be described with

$$
A(t)=A_{0}\left[1-e^{t / \tau}\right],
$$

where $\tau$ is the time constant of the recovery time. We measured the recovery time using the latter method. The black dots in Fig. 19 are afterpulses selected to be fit with the above function, which is shown as the solid black line in the figure.

The measured recovery time constants are shown in Fig. 29 for all devices. At the operating voltages, the time constants are in good agreement with the product of the cell capacitance and quench resistors.

An expected trend that is observed for all devices is the decrease of the recovery time with increasing temperature, which is due to the decreasing value of the quench resistor. (s. Fig. 15).

\section{Discussion}

In this paper we presented the characterization of three 222 recent, blue-sensitive SiPMs from FBK, Hamamatsu, and ${ }_{223}$ SensL. All three devices show superior performance $\mathrm{in}_{224}$ terms of their optical and electrical characteristics with 225 respect to past generations of SiPMs.

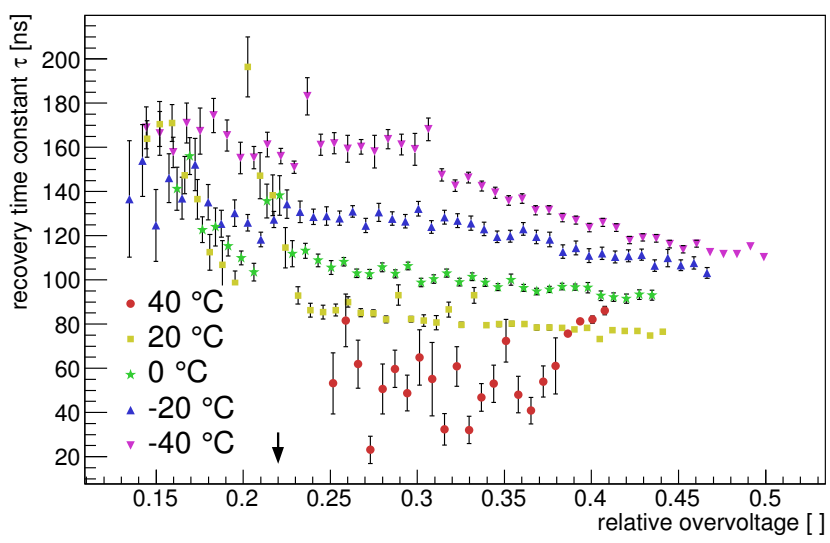

(a) FBK NUV-HD

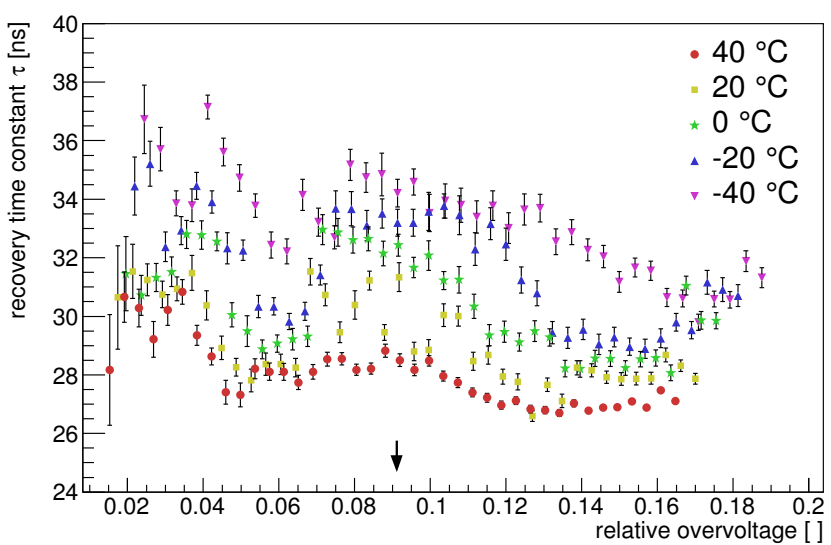

(b) Hamamatsu S13360-3050CS

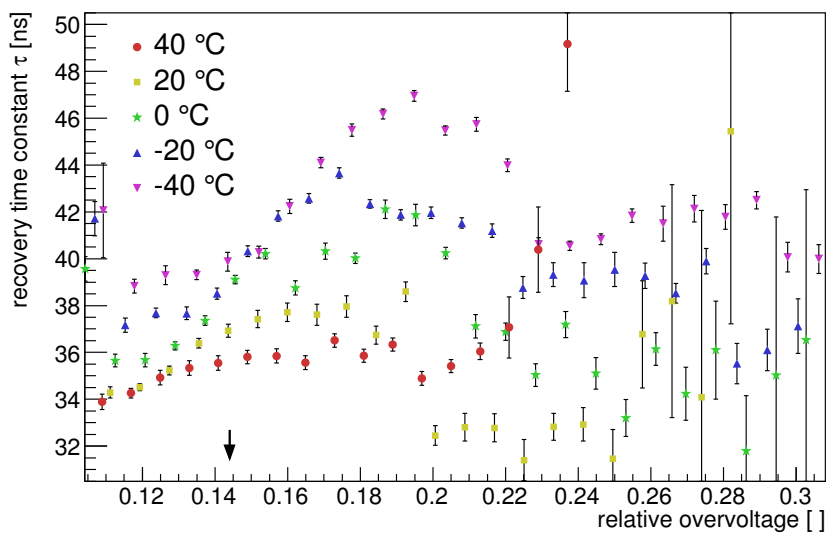

(c) SensL J-series 30035

Figure 29: Recovery times. The arrow marks the nominal operating bias of each device.

The very good performance of the three devices motivated us to investigate how to best parameterize SiPM characteristics as a function of bias and temperature. We believe that standardizing the parameterization of SiPMs will become increasingly important as the community of SiPM users is constantly growing, and not everyone has inhouse capabilities to perform in-depth device studies. Furthermore, the optimal operating point of an SiPM varies 
from application to application, which requires knowledge284 of SiPM parameters over a wide range of temperature 285 and bias. With a standardized SiPM parameterization atz28 hand, the user can focus on the application and with the 287 help of the model find the optimal $\mathrm{SiPM}$ and its operating $\mathrm{g}_{28}$ point.

We have found parameterizations of the breakdown 290 probability, optical crosstalk, dark rate, and afterpulsing 291 as a function of temperature and bias that can be applied222 to all three tested SiPMs. The parameterization also al ${ }_{+293}$ lows extraction of physical parameters like the location of $2_{294}$ the high-field region using $\alpha$ in the breakdown probability ${ }_{\mathbf{3} 295}$ or the optical crosstalk efficiency factor $\gamma$.

The choice of comparing device characteristics at the 297 bias where the PDE at $400 \mathrm{~nm}$ reaches $90 \%$ breakdown 298 probability is driven by our ultimate desire to obtain 299 SiPMs with the highest optical efficiency and, at the same 300 time, sufficiently low nuisance parameters. If one has tq301 select one of the three devices for an application, detailed 302 end-to-end simulations are needed that find the bias that ${ }_{303}$ results in the best compromise between PDE and nuisance ${ }_{304}$ parameters. Such a study is not within the scope of this $\$_{305}$ paper. Instead we discuss how well the tested device ${ }_{306}$ match the requirements for Cherenkov telescopes when ${ }_{307}$ the SiPMs are operated at $90 \%$ breakdown probability ${ }_{1308}$ and we point out the remaining shortcomings that prevent ${ }_{309}$ the tested devices from being perfect photon detectors for ${ }_{1310}$ Cherenkov telescopes when operated at that bias.

Reduced optical crosstalk, afterpulsing, and dark-count rates allow the operation of all three devices at much $_{313}$ higher relative overvoltages, thus yielding breakdown $\eta_{314}$ probabilities of more than $90 \%$ for blue photons. Not only $y_{1315}$ does a $90 \%$ breakdown probability provide a significant boost in PDE, but it also reduces the sensitivity of gain ${ }_{316}$ and PDE on temperature changes. Using that one $\mathrm{de}_{1317}$ gree change in temperature shifts the breakdown voltage ${ }_{318}$ by $0.1 \%$ for all three devices; the gain of an SiPM changes ${ }_{319}$ by $1 \% /{ }^{\circ} \mathrm{C}$ if it is operated at $10 \%$ overvoltage. If a de $\mathrm{de}_{\text {T320 }}$ vice is operated at $20 \%$ overvoltage, the gain changes by $\mathrm{y}_{321}$ only $0.5 \% /{ }^{\circ} \mathrm{C}$. The three tested devices operate in between ${ }_{322}$ these limits.

The temperature dependence of the PDE is even smalle $\boldsymbol{H}_{324}$ because the breakdown probability is in saturation. With $\mathrm{W}_{325}$ our parameterization of the breakdown probability it can be calculated that the relative PDE changes between 326 $0.2 \% /{ }^{\circ} \mathrm{C}$ and $0.3 \% /{ }^{\circ} \mathrm{C}$ for the three tested devices if they 327 are operated at $90 \%$ breakdown probability. These values 328 are on par with typical values for bialkali photomultiplier 329 tubes [24]. Measures to temperature-stabilize SiPMs in 330 applications or to correct data offline is, therefore, not331 necessary anymore, or the requirements to temperature ${ }_{+332}$ stabilize devices can be much more relaxed.

The peak PDE of the three devices ranges between $40 \% 334$ and $50 \%$, which, again, is a huge improvement compared 335 to the PDEs of devices available just 10 years ago. Being 336 able to operate at $90 \%$ breakdown probability is certainly 333 one main reason for the high PDEs, but it is worth noting ${ }_{338}$ that the spectral response has shifted considerably into the blue/UV region. Considering that the maximum achievable geometrical fill factor is probably around $80 \%$, the maximum possible PDE that can be expected for SiPMs is around $65 \%$ assuming a $90 \%$ breakdown probability and a $90 \%$ quantum efficiency. In fact, FBK recently presented results of SiPMs with a peak PDE of more than $60 \%$ PDE [25]. Enhancing the blue efficiency of SiPMs further and shifting their peak efficiency toward lower wavelengths is likely to be realized by thinning the passivation layer and the first implant, which will be technological challenges.

Optical crosstalk, dark rates, and afterpulsing are also much reduced in comparison to older devices. Dark rates are typically a few ten $\mathrm{kHz} / \mathrm{mm}^{2}$, whereas early devices typically had rates of one $\mathrm{MHz} / \mathrm{mm}^{2}$. Optical crosstalk has been lowered by reducing cell capacitances, introducing trenches between cells, and optimizing the layout of structures. Each tested devices has successfully implemented one or more of the aforementioned measures, and direct optical crosstalk ranges between $6 \%$ and $20 \%$ at $90 \%$ breakdown probability.

Delayed optical crosstalk and afterpulsing are two more nuisance parameters that could be considerably improved, with typical values being $\sim 2 \%$.

Parameters that are well within the requirements are cell recovery time and gain. A lower gain and a smaller cell recovery time in future devices is perfectly acceptable. A lower gain would reduce power dissipated by the SiPM, which is a plus when SiPMs are used in environments with intense photon backgrounds.

Given all of these improvements, only a short list of desirable changes remain:

- The sensitivity should be highest between $250 \mathrm{~nm}$ and $550 \mathrm{~nm}$ if possible with a flat response. Above $550 \mathrm{~nm}$ the sensitivity should cut off sharply. Such a spectral response would maximize the detection of Cherenkov light and at the same time efficiently reject ambient light coming from the night sky, which dominates at long wavelengths. Of the three tested devices, the FBK device comes closest to the ideal response, but improvements would still be desirable to further suppress the response at long wavelengths.

- Direct optical crosstalk is one of the main factors limiting the lowest achievable trigger threshold. The majority of trigger concepts used in Cherenkov telescopes employ an $\mathrm{n}$-fold coincidence of neighboring camera pixels. In the coincidence, each pixel has to have a signal above a certain threshold. How low that threshold can be set depends ideally only on the maximum acceptable trigger rate due to statistical up-fluctuations in the ambient light. For most operating or planned Cherenkov telescopes, a direct optical crosstalk of $3 \%$ would double that trigger rate which would be acceptable. It is of course desirable to minimize optical crosstalk as much as possible. With $6 \%$ optical 
crosstalk, the Hamamatsu device is not far from an 392 optimal value.

- Afterpulsing and delayed optical crosstalk add to the $e^{1395}$ effective dark-count rate and contaminate the $\operatorname{ex}_{\overline{1} 397}^{1396}$ tracted Cherenkov signal by introducing a positive 398 bias. With about $2 \%$ afterpulsing and delayed optical ${ }^{399}$ crosstalk, respectively, all three devices have accept ${ }_{1401}^{1400}$ able values that can be dealt with at the stage of $\operatorname{sig}_{1402}$ nal extraction. However, keeping both effects below 403 $1 \%$ would simplify the data analysis and reduce sys ${ }^{1404}$ tematic uncertainties in the energy scale of Cherenkov $v_{1406}^{1405}$ telescopes.

- The cost of SiPMs is still a dominant contribution t $\sigma^{409}$ the total per channel costs (readout electronics and $d^{410}$ photosensor). Considerable efforts have been made $i_{412}^{1411}$ the past to reduce the cost of the readout electronics \$ $_{\$ 413}$ and it is not unreasonable to assume that with new ${ }^{414}$ concepts costs of $\$ 5$ per readout channel can be real ${ }_{1416}^{1415}$ ized in the future. SiPMs would have to cost about 1416 $\$ 0.1 / \mathrm{mm}^{2}$ to contribute equally to the per channel ${ }_{418}$ costs.

1419

All these items are major technological challenges, but it is ${ }^{1421}$ not evident that fundamental physical limitations preclude $e_{423}^{1422}$ one from surmounting them. Therefore, we are confident 424 that new and improved devices will become available $\operatorname{ir}^{425}$ the future.

\section{Acknowledgment}

We are grateful to FBK, Hamamatsu, and SensL for ${ }^{1432}$ providing us with samples of their latest developments ${ }_{1434}$ We thank J. Biteau for useful discussions and input tha $\star_{435}$ improved the extraction of the breakdown voltage from ${ }^{436}$ the IV curves. in our procedure to fit the IV curves This ${ }^{1437}$ research was in part supported by the National Science S3 $_{439}$ Foundation under grant no. PHYS-1505228.

\section{References}

[1] A. N. Otte, K. Meagher, T. Nguyen, M. Carroll, S. Hooper ${ }_{1446}^{1445}$ K. McKinney, S. Peet, Silicon photomultiplier integration in the $\mathrm{I}_{447}$ camera of the mid-size Schwarzschild-Couder Cherenkov tele $\overline{\overline{1}}_{\overline{1} 448}$ scope for CTA, Nuclear Instruments and Methods in Physic\$ ${ }_{149}$ Research A 787 (2015) 85-88. doi:10.1016/j.nima.2014.11.026. . $_{1450}$

[2] B. Lutz, CMS Collaboration, Upgrade of the CMS Hadroh 450 Outer Calorimeter with SiPM sensors, Journal of Physic\$ $\$_{452}$ Conference Series 404 (1) (2012) 012018. doi:10.1088/1742 ${ }_{\overline{1} 453}$ $6596 / 404 / 1 / 012018$.

[3] Y. Sun, J. Maricic, SiPMs characterization and selection ${ }_{4455}^{1454}$ for the DUNE far detector photon detection system, Jour ${ }_{\overline{14} 56}$ nal of Instrumentation 11 (2016) C01078. arXiv:1511.04756 $6_{1457}$ doi:10.1088/1748-0221/11/01/C01078.

[4] A. Benaglia, S. Gundacker, P. Lecoq, M. T. Lucchini, A. Para ${ }_{1459}^{145}$ K. Pauwels, E. Auffray, Detection of high energy muons with $_{460}$ sub-20 ps timing resolution using L(Y)SO crystals and $\mathrm{SiPM}_{461}$ readout, Nuclear Instruments and Methods in Physics Research ${ }_{462}$ A 830 (2016) 30-35. doi:10.1016/j.nima.2016.05.030.
[5] E. Lorenz, R. Wagner, Very-high energy gamma-ray astronomy. A 23-year success story in high-energy astroparticle physics, European Physical Journal H 37 (2012) 459-513. arXiv:1207.6003, doi:10.1140/epjh/e2012-30016-x.

[6] B. Dolgoshein, V. Balagura, P. Buzhan, M. Danilov, L. Filatov, E. Garutti, M. Groll, A. Ilyin, V. Kantserov, V. Kaplin, A. Karakash, F. Kayumov, S. Klemin, V. Korbel, H. Meyer, R. Mizuk, V. Morgunov, E. Novikov, P. Pakhlov, E. Popova, V. Rusinov, F. Sefkow, E. Tarkovsky, I. Tikhomirov, Calice/SiPM Collaboration, Status report on silicon photomultiplier development and its applications, Nuclear Instruments and Methods in Physics Research A 563 (2006) 368-376. doi:10.1016/j.nima.2006.02.193.

[7] D. Renker, E. Lorenz, Advances in solid state photon detectors, Journal of Instrumentation 4 (2009) 04004. doi:10.1088/17480221/4/04/P04004.

[8] C. Piemonte, F. Acerbi, A. Ferri, A. Gola, G. Paternoster, V. Regazzoni, G. Zappala, N. Zorzi, Performance of NUV-HD Silicon Photomultiplier Technology, IEEE Transactions on Electron Devices 63 (2016) 1111-1116. doi:10.1109/TED.2016.2516641.

[9] B. S. Acharya, M. Actis, T. Aghajani, G. Agnetta, J. Aguilar, F. Aharonian, M. Ajello, A. Akhperjanian, M. Alcubierre, J. Aleksić, et al., Introducing the CTA concept, Astroparticle Physics 43 (2013) 3-18. doi:10.1016/j.astropartphys.2013.01.007.

[10] S13360-3050cs datasheet.

URL http://www.hamamatsu.com/resources/pdf/ssd/s13360_ series_kapd1052e.pdf

[11] Sensl j-series datasheet. URL http://sensl.com/products/j-series/

[12] A. N. Otte, J. Hose, R. Mirzoyan, A. Romaszkiewicz, M. Teshima, A. Thea, A measurement of the photon detection efficiency of silicon photomultipliers, Nuclear Instruments and Methods in Physics Research A 567 (2006) 360-363. doi:10.1016/j.nima.2006.05.145.

[13] Hamamatsu Photonics K. K., private communication (2016).

[14] R. Gunnink, et. al., Gamma-Ray Energies and Intensities, UCID-15439.

[15] C. Xu, Study of the Silicon Photomultipliers and Their Applications in Positron Emission Tomography, Ph.D. thesis, Universität Hamburg (2014).

[16] Y. Musienko, Radiation Damage Studies of Silicon Photomultipliers for the CMS HCAL Phase I Upgrade, "NDIP 2104" (2014).

URL http://ndip.in2p3.fr/ndip14/AGENDA/AGENDA-by-DAY/ Presentations/5Friday/PM/ID37166_Musienko.pdf

[17] N. Serra, G. Giacomini, A. Piazza, C. Piemonte, A. Tarolli, N. Zorzi, Experimental and TCAD Study of Breakdown Voltage Temperature Behavior in $\mathrm{n}^{\wedge}\{+\} / \mathrm{p}$ SiPMs, IEEE Transactions on Nuclear Science 58 (2011) 1233-1240. doi:10.1109/TNS.2011.2123919.

[18] C. Piemonte, R. Battiston, M. Boscardin, G.-F. Dalla Betta, A. Del Guerra, N. Dinu, A. Pozza, N. Zorzi, Characterization of the First Prototypes of Silicon Photomultiplier Fabricated at ITC-irst, IEEE Transactions on Nuclear Science 54 (2007) 236-244. doi:10.1109/TNS.2006.887115.

19] C. Piemonte, A. Ferri, A. Gola, A. Picciotto, T. Pro, N. Serra, A. Tarolli, N. Zorzi, Development of an automatic procedure for the characterization of silicon photomultipliers, in: Proceedings, 2012 IEEE Nuclear Science Symposium and Medical Imaging Conference (NSS/MIC 2012): Anaheim, California, USA, October 29-November 3, 2012, 2012, pp. 428-432. doi:10.1109/NSSMIC.2012.6551141.

[20] ROOT data analysis framework. URL http://root.cern.ch

[21] A. N. Otte, On the efficiency of photon emission during electrical breakdown in silicon, Nuclear Instruments and Methods in Physics Research Section A: Accelerators, Spectrometers, Detectors and Associated Equipment 610 (1) (2009) 105 - 109, new Developments In Photodetection \{NDIP08Proceedings of the 
Fifth International Conference on New Developments in Photodetection. doi:http://dx.doi.org/10.1016/j.nima.2009.05.085.

URL http://www.sciencedirect.com/science/article/pii/ S0168900209010390

[22] J. Rosado, V. Aranda, F. Blanco, F. Arqueros, Modeling crosstalk and afterpulsing in silicon photomultipliers, Nuclear Instruments and Methods in Physics Research Section A: Accelerators, Spectrometers, Detectors and Associated Equipment 787 (2015) 153 - 156, new Developments in Photodetection \{NDIP14\}. doi:http://dx.doi.org/10.1016/j.nima.2014.11.080. URL http://www.sciencedirect.com/science/article/pii/ S0168900214013862

[23] A. Gola, A. Ferri, A. Tarolli, N. Zorzi, C. Piemonte, Sipm optical crosstalk amplification due to scintillator crystal: effects on timing performance, Physics in Medicine and Biology 59 (13) (2014) 3615 .

URL http://stacks.iop.org/0031-9155/59/i=13/a=3615

24] Hamamatsu Photonics K. K., PHOTOMULTIPLIER TUBES Basics and Applications V3a (2007).

URL http://www.hamamatsu.com/resources/pdf/etd/PMT_ handbook_v3aE.pdf

[25] C. Piemonte, private communication (2016). 